\title{
INVERSE PROBLEM FOR THE HEAT EQUATION AND THE SCHRÖDINGER EQUATION ON A TREE
}

\author{
LIVIU I. IGNAT, ADEMIR F. PAZOTO, AND LIONEL ROSIER
}

\begin{abstract}
In this paper we establish global Carleman estimates for the heat and Schrödinger equations on a network. The heat equation is considered on a general tree and the Schrödinger equation on a star-shaped tree. The Carleman inequalities are used to prove the Lipschitz stability for an inverse problem consisting in retrieving a stationary potential in the heat (resp. Schrödinger) equation from boundary measurements.
\end{abstract}

\section{INTRODUCTION}

In this paper we consider two inverse problems on a network formed by the edges of a tree. The problems we address here enter in the framework of quantum graphs. The name quantum graph is used for a graph considered as a one-dimensional singular variety and equipped with a differential operator. Those quantum graphs are metric spaces which can be written as the union of finitely many intervals, which are compact or $[0, \infty)$ and any two of these intervals are either disjoint or intersect only at one of their endpoints.

Quantum graphs arise as simplified models in mathematics, physics, chemistry, and engineering (e.g., nanotechnology and microelectronics), when one considers propagation of waves through a quasi-one-dimensional system that looks like a thin neighborhood of a graph. We can mention in particular the quantum wires and thin waveguides. Differential operators on metric graphs arise in a variety of applications, to quote a few: carbon nano-structures [28], photonic crystals [16], high-temperature granular superconductors [1], quantum waveguides [12], freeelectron theory of conjugated molecules in chemistry, quantum chaos, etc. For more details we refer the reader to the review papers [25], [27], [26], [15] and the references therein for more informations on this topic.

To be more precise we consider the heat equation on a 1-D network $\Gamma$ given by the edges of a general tree and the Schrödinger equation on a star-shaped tree.

The first system we consider is the following one

$$
\begin{cases}\mathbf{u}_{t}-\Delta_{\Gamma} \mathbf{u}+\mathbf{p u}=0, & \text { in } \Gamma \times(0, T), \\ \mathbf{u}=\mathbf{h}, & \text { on } \partial \Gamma \times(0, T), \\ \mathbf{u}(\cdot, 0)=\mathbf{u}_{0}, & \text { in } \Gamma,\end{cases}
$$

where $\Delta_{\Gamma}$ is the Laplace operator on the network $\Gamma$. The system is closed with the coupling conditions at the internal nodes of the tree, namely the continuity and the Kirchhoff's law on the

Key words and phrases. Carleman estimate; tree; network; heat equation; Schrödinger equation; inverse problem. 
flux at all internal vertices of $\Gamma$. Here, $\mathbf{u}$ is a collection of functions $u^{\bar{\alpha}}$ each of them satisfying a heat equation on some edge of the network.

Simultaneously with problem (1.1) we consider the following problem

$$
\begin{cases}i \mathbf{u}_{t}+\Delta_{\Gamma} \mathbf{u}+\mathbf{p u}=0, & \text { in } \Gamma \times(0, T), \\ \mathbf{u}=\mathbf{h}, & \text { on } \partial \Gamma \times(0, T), \\ \mathbf{u}(\cdot, 0)=\mathbf{u}_{0}, & \text { in } \Gamma,\end{cases}
$$

under similar coupling conditions as in the previous model.

In both cases we are interested in determining the potential $\mathbf{p}$, a collection of functions defined on the edges of $\Gamma$, from boundary measurements. In the case of the first system, we are able to prove that we can recover $\mathbf{p}$ using only $N-1$ measurements, where $N$ is the total number of exterior nodes of the network $\Gamma$. However, in the case of the second system, besides of the fact that we need to deal with a star-shaped network, we only can recover the potential $\mathbf{p}$ from measurements performed at all the exterior nodes of $\Gamma$.

The use of Carleman estimates to achieve uniqueness and stability results in inverse problems is well known. Some authors use local Carleman inequalities and deduce uniqueness and Hölder estimates. Others make use of global Carleman inequalities and deduce Lipschitz stability results and hence uniqueness results. We shall follow that second approach.

Inverse problems with a finite number of measurements have been widely studied by Bukhgeim and Klibanov (see [8], [21], and [22]) by means of Carleman estimates (see also the book [20] and the references therein). For a wide class of partial differential equations, their method provides the stability in the inverse problem, whenever a suitable Carleman estimate is available. Since [8], there have been many works based upon their methodology.

The theory of global Carleman estimates for parabolic operator has been largely developed since the work by Fursikov-Imanuvilov [17] and it has been applied to many situations (e.g. to prove the controllability along the trajectories or the stability in inverse problems). Since a complete list of references is too long we refer the reader to [34] for a quite complete review of the state of art.

Concerning the Schrödinger equation we refer to $[4,6,9,10,30]$ where Carleman estimates are proved and used to establish the stability for some inverse problems (see also [19, 29] for some other Carleman estimates for Schrödinger equation).

The same approach has given many results for the wave equation. Since a complete list is too long we quote only some of them, related to the same inverse problem consisting in retrieving a stationary potential in wave equation: [31] and [33] for Dirichlet boundary data and a Neumann measurement and [18] for Neumann boundary data and a Dirichlet measurement. These references are based on the use of local or global Carleman estimates. In the framework of Carleman inequalities on networks we mention the recent paper [3] where the authors establish a global Carleman estimate for the wave equation on a star-shaped tree and used it to derive the Lipschitz stability in an inverse problem. The Carleman estimate in [3] involves some positive definite matrix introduced in [7] to derive a Carleman estimate for the one-dimensional heat equation with discontinuous coefficients. 
As far as we know, the determination of a time-independent potential for the heat or Schrödinger equation in a network-like structure has not been addressed in the literature yet. This type of problems has been studied for example for membranes or elastic strings (see for instance [2] and the references therein).

Let us now state the main results of the paper. For a given initial data $\mathbf{u}_{0}$ and a given boundary data $\mathbf{h}$, we denote by $\mathbf{u}(\mathbf{p})$ the solution of the above systems associated with the potential $\mathbf{p} \in L^{\infty}(\Gamma, \mathbb{R})$. We introduce the space

$$
H^{2,1}(\Gamma \times(0, T)):=L^{2}\left(0, T ; H^{2}(\Gamma)\right) \cap H^{1}\left(0, T ; L^{2}(\Gamma)\right) .
$$

(See below Section 2 for the definition of $H^{2}(\Gamma)$.) We also introduce the ball $B_{m}(0):=\{\mathbf{q} \in$ $\left.L^{\infty}(\Gamma, \mathbb{R}) ;\|\mathbf{q}\|_{L^{\infty}(\Gamma)} \leq m\right\}$. Then the following stability results hold.

Theorem 1.1. Assume that $\mathbf{p} \in L^{\infty}(\Gamma), \mathbf{u}_{0}=\mathbf{u}_{0}(x), h=h(x, t)$ and $r>0$ are such that the solution $\mathbf{u}(\mathbf{p})$ of (1.1) fulfills $\mathbf{u}(\mathbf{p}) \in H^{2,1}(\Gamma \times(0, T)), \partial_{t} \mathbf{u}(\mathbf{p}) \in H^{2,1}(\Gamma \times(0, T))$, and such that for some $t_{0} \in(0, T)$ it holds

$$
\left|\mathbf{u}(\mathbf{p})\left(\cdot, t_{0}\right)\right| \geq r \text { a.e. on } \Gamma \text {. }
$$

Then, for any $m>0$ there exists a constant $C=C\left(m,\left\|\partial_{t} \mathbf{u}(\mathbf{p})\right\|_{L^{\infty}(\Gamma \times(0, T))}, r\right)$ such that for any $\mathbf{q} \in B_{m}(0)$ satisfying

$$
\partial_{x}[\mathbf{u}(\mathbf{p})-\mathbf{u}(\mathbf{q})](v, .) \in H^{1}(0, T) \text { for all exterior nodes } v,
$$

we have

$$
\begin{aligned}
& \|\mathbf{p}-\mathbf{q}\|_{L^{2}(\Gamma)} \\
& \quad \leq C\left(\left\|[\mathbf{u}(\mathbf{p})-\mathbf{u}(\mathbf{q})]\left(\cdot, t_{0}\right)\right\|_{H^{2}(\Gamma)}+\sum_{v \in \mathcal{E}}\left\|\partial_{x}[\mathbf{u}(\mathbf{p})-\mathbf{u}(\mathbf{q})](v, \cdot)\right\|_{H^{1}(0, T)}\right),
\end{aligned}
$$

where $\mathcal{E}$ denotes the set of all the exterior vertices of $\Gamma$ except one.

For the second system, under the assumption that the network is a star-shaped tree, we can prove a similar stability result.

Theorem 1.2. Assume that $\mathbf{p} \in L^{\infty}(\Gamma ; \mathbb{R}), \mathbf{u}_{0}=\mathbf{u}_{0}(x), h=h(x, t)$ and $r>0$ are such that the solution of (1.2) satisfies

- $\mathbf{u}_{0}(x) \in \mathbb{R}$ or $i \mathbf{u}_{0}(x) \in \mathbb{R}$ a.e. in $\Gamma$,

- $\left|\mathbf{u}_{0}(x)\right| \geq r>0$ a.e. in $\Gamma$, and

- $\partial_{t} \mathbf{u}(\mathbf{p}) \in H^{2,1}(\Gamma \times(0, T))$.

Then, for any $m \geq 0$, there exists a constant $C=C\left(m,\left\|\partial_{t} \mathbf{u}(\mathbf{p})\right\|_{H^{2,1}(\Gamma \times(0, T))}, r\right)>0$ such that for any $\mathbf{q} \in B_{m}(0)$ satisfying

$$
\partial_{t} \mathbf{u}(\mathbf{q}) \in H^{2,1}(\Gamma \times(0, T))
$$

we have

$$
\|\mathbf{p}-\mathbf{q}\|_{L^{2}(\Gamma)} \leq C \sum_{v \in \partial \Gamma}\left\|\partial_{x}[\mathbf{u}(\mathbf{p})-\mathbf{u}(\mathbf{q})](v, .)\right\|_{H^{1}(0, T)}{ }^{*}
$$


The above theorems extend to networks classical results on inverse problems. To prove those results, we need to establish (new) global Carleman estimates for the heat (resp. the Schrödinger) equation on trees. Note that if we impose Kirchhoff-type conditions to the weight function at the internal vertices, the Carleman estimate cannot be derived. In our Carleman estimates, the weight function has to fulfill some nonlinear flux condition at each internal vertex. On the other hand, for the Schrödinger equation posed on a star-shaped tree with $N$ external vertices, we consider a combination of $N$ weight functions in order to cancel some "bad" terms at the internal vertices involving time derivatives. That strategy was used in [5], with two different weight functions, in order to improve the observation region for the wave equation.

The article is organized as follows. In Section 2 we introduce the notations and some classical facts about the heat and Schrödinger equations on trees. Section 3 presents the analysis in the case of the heat equation. The Schrödinger equation is considered in Section 4. Finally we discuss some open problems in Section 5.

\section{Notations And Preliminaries}

Let $\Gamma=(V, E)$ be a graph where $V$ is the set of vertices and $E$ the set of edges. The edges are assumed to be of finite length and their ends are the vertices of $V$. For each $v \in V$ we denote $E_{v}=\{e \in E: v \in e\}$. The multiplicity of a vertex of $\Gamma$ is equal to the number of edges that branch out from it. If the multiplicity is equal to one, the vertex is said to be exterior, otherwise it is said to be interior. We assume that $\Gamma$ does not contain vertices with multiplicity two, since they are irrelevant for our models.

From now on, we assume that $\Gamma$ is a tree, that is, $\Gamma$ is a planar finite connected graph without circuit (closed path). We fix an orientation of $\Gamma$ and for each oriented edge $e$, we denote by $I(e)$ its initial vertex and by $T(e)$ its terminal one.

We identify every edge $e$ of $\Gamma$ with an interval $I_{e}$, where $I_{e}=\left[0, l_{e}\right], l_{e}$ being the length of $e$. This identification introduces a coordinate $x_{e}$ along the edge $e$.

Let $v$ be a vertex of $V$ and $e$ be an edge in $E_{v}$. We set

$$
i(v, e)= \begin{cases}0 & \text { if } \quad v=I(e), \\ l_{e} & \text { if } \quad v=T(e) .\end{cases}
$$

We identify any function $\mathbf{u}$ on $\Gamma$ with a collection $\left\{u^{e}\right\}_{e \in E}$ of functions $u^{e}$ defined on the edges $e$ of $\Gamma$. Each $u^{e}$ can be considered as a function on the interval $I_{e}$. In fact, we use the same notation $u^{e}$ for both the function on the edge $e$ and the function on the interval $I_{e}$ identified with $e$. For a function $\mathbf{u}: \Gamma \rightarrow \mathbb{C}, \mathbf{u}=\left\{u^{e}\right\}_{e \in E}$, we denote by $f(\mathbf{u}): \Gamma \rightarrow \mathbb{C}$ the family $\left\{f\left(u^{e}\right)\right\}_{e \in E}$, where $f\left(u^{e}\right): e \rightarrow \mathbb{C}$.

A function $\mathbf{u}=\left\{u^{e}\right\}_{e \in E}$ is continuous if and only if $u^{e}$ is continuous on $I_{e}$ for every $e \in E$, and $\mathbf{u}$ is continuous at the vertices of $\Gamma$ :

$$
u^{e}(i(v, e))=u^{e^{\prime}}\left(i\left(v, e^{\prime}\right)\right), \quad \forall e, e^{\prime} \in E_{v} .
$$

The space $L^{p}(\Gamma), 1 \leq p<\infty$ consists of all the functions $\mathbf{u}=\left\{u_{e}\right\}_{e \in E}$ on $\Gamma$ that belong to $L^{p}\left(I_{e}\right)$ for each edge $e \in E$. That space is endowed with the norm

$$
\|\mathbf{u}\|_{L^{p}(\Gamma)}^{p}=\sum_{e \in E}\left\|u^{e}\right\|_{L^{p}\left(I_{e}\right)}^{p}<\infty
$$


Similarly, the space $L^{\infty}(\Gamma)$ consists of all the functions $\mathbf{u}=\left\{u_{e}\right\}_{e \in E}$ that belong to $L^{\infty}\left(I_{e}\right)$ for each edge $e \in E$. The corresponding norm is

$$
\|\mathbf{u}\|_{L^{\infty}(\Gamma)}=\sup _{e \in E}\left\|u^{e}\right\|_{L^{\infty}\left(I_{e}\right)}<\infty .
$$

The Sobolev space $H^{m}(\Gamma)$, with $m \in \mathbb{N}^{*}$, consists of all the continuous functions on $\Gamma$ that belong to $H^{m}\left(I_{e}\right)$ for each $e \in E$. It is endowed with the norm

$$
\|\mathbf{u}\|_{H^{m}(\Gamma)}^{2}=\sum_{e \in E}\left\|u^{e}\right\|_{H^{m}(e)}^{2}<\infty .
$$

The spaces $L^{2}(\Gamma)$ and $H^{m}(\Gamma)$ are Hilbert spaces when endowed with the inner products

$$
(\mathbf{u}, \mathbf{v})_{L^{2}(\Gamma)}=\sum_{e \in E}\left(u^{e}, v^{e}\right)_{L^{2}\left(I_{e}\right)}=\sum_{e \in E} \int_{I_{e}} u^{e}(x) \overline{v^{e}}(x) d x
$$

and

$$
(\mathbf{u}, \mathbf{v})_{H^{m}(\Gamma)}=\sum_{e \in E}\left(u^{e}, v^{e}\right)_{H^{m}\left(I_{e}\right)}=\sum_{e \in E} \sum_{k=0}^{m} \int_{I_{e}} \frac{d^{k} u^{e}}{d x^{k}} \frac{\overline{d^{k} v^{e}}}{d x^{k}} d x .
$$

$H_{0}^{1}(\Gamma)$ denotes the set of functions in $H^{1}(\Gamma)$ that vanish at the exterior vertices. We now introduce the Laplace operator $\Delta_{\Gamma}$ on the tree $\Gamma$. Even if it is a standard procedure, we prefer to recall it following [11], for the sake of completeness. Consider the sesquilinear continuous form $\varphi$ on $H_{0}^{1}(\Gamma)$ defined by

$$
\varphi(\mathbf{u}, \mathbf{v})=\left(\mathbf{u}_{x}, \mathbf{v}_{x}\right)_{L^{2}(\Gamma)}=\sum_{e \in E} \int_{I_{e}} u_{x}^{e}(x) \overline{v_{x}^{e}}(x) d x .
$$

We denote by $D\left(\Delta_{\Gamma}\right)$ the set of all the functions $\mathbf{u} \in H_{0}^{1}(\Gamma)$ such that the linear map $\mathbf{v} \in$ $H_{0}^{1}(\Gamma) \rightarrow \varphi_{\mathbf{u}}(\mathbf{v}):=\varphi(\mathbf{u}, \mathbf{v})$ satisfies

$$
\left|\varphi_{\mathbf{u}}(\mathbf{v})\right| \leq C\|\mathbf{v}\|_{L^{2}(\Gamma)} \quad \text { for all } \mathbf{v} \in H_{0}^{1}(\Gamma) .
$$

For $\mathbf{u} \in D\left(\Delta_{\Gamma}\right)$, we can extend $\varphi_{\mathbf{u}}$ to a linear continuous mapping on $L^{2}(\Gamma)$. There is a unique element in $L^{2}(\Gamma)$, denoted by $\Delta_{\Gamma} \mathbf{u}$, such that

$$
\varphi(\mathbf{u}, \mathbf{v})=-\left(\Delta_{\Gamma} \mathbf{u}, \mathbf{v}\right)_{L^{2}(\Gamma)} \quad \text { for all } \mathbf{v} \in H_{0}^{1}(\Gamma) .
$$

We now define the normal exterior derivative of a function $\mathbf{u}=\left\{u^{e}\right\}_{e \in E}$ at the endpoints of the edges. For each $e \in E$ and $v$ an endpoint of $e$ we consider the normal derivative of the restriction of $\mathbf{u}$ to the edge $e$ of $E_{v}$ evaluated at $i(v, e)$ to be defined by:

$$
\frac{\partial u^{e}}{\partial n_{e}}(i(v, e))= \begin{cases}-u_{x}^{e}(0+) & \text { if } \quad i(v, e)=0 \\ u_{x}^{e}\left(l_{e}-\right) & \text { if } \quad i(v, e)=l_{e} .\end{cases}
$$

With this notation it is easy to characterize $D\left(\Delta_{\Gamma}\right)$ (see [11]):

$$
D\left(\Delta_{\Gamma}\right)=\left\{\mathbf{u}=\left\{u^{e}\right\}_{e \in E} \in H^{2}(\Gamma) \cap H_{0}^{1}(\Gamma) ; \sum_{e \in E_{v}} \frac{\partial u^{e}}{\partial n_{e}}(i(v, e))=0 \quad \text { for any interior vertex } v\right\}
$$

and

$$
\left(\Delta_{\Gamma} \mathbf{u}\right)^{e}=\left(u^{e}\right)_{x x} \quad \text { for all } e \in E, \mathbf{u} \in D\left(\Delta_{\Gamma}\right)
$$


In other words $D\left(\Delta_{\Gamma}\right)$ is the space of all the continuous functions $\mathbf{u}=\left\{u^{e}\right\}_{e \in E}$ on $\Gamma$, such that for each edge $e \in E, u^{e} \in H^{2}\left(I_{e}\right)$, and which vanish at each exterior node and fulfill the following Kirchhoff-type condition

$$
\sum_{e \in E ; T(e)=v} u_{x}^{e}\left(l_{e}-\right)-\sum_{e \in E ; I(e)=v} u_{x}^{e}(0+)=0
$$

at each interior node $v$. It is easy to verify that $\left(\Delta_{\Gamma}, D\left(\Delta_{\Gamma}\right)\right)$ is a linear, unbounded, self-adjoint, dissipative operator on $L^{2}(\Gamma)$, i.e. $\Re\left(\Delta_{\Gamma} \mathbf{u}, \mathbf{u}\right)_{L^{2}(\Gamma)} \leq 0$ for all $\mathbf{u} \in D\left(\Delta_{\Gamma}\right)$.

\section{The heAT EQUATION}

3.1. Preliminaries and notations. In this section we introduce the notations for the elements of the considered tree. We mainly follow the notations of [14].

We first describe the procedure to index the edges and vertices of the tree. We first choose an exterior vertex, called the root of the tree and denoted by $\mathcal{R}$. The remaining edges and vertices will be denoted by $e_{\bar{\alpha}}$ and $\mathcal{O}_{\bar{\alpha}}$, respectively, where $\bar{\alpha}=\left(\alpha_{1}, \ldots, \alpha_{k}\right)$ is a multi-index (taking value in $\{1\} \cup \bigcup_{k \geq 2} \mathbb{N}^{k}$ ). The multi-indices are defined by induction in the following way. For the edge containing the root $\mathcal{R}$ we choose the index 1 . That edge is denoted by $e_{\overline{1}}$ and its second end is denoted by $\mathcal{O}_{\overline{1}}$. Assume now that the interior vertex $\mathcal{O}_{\bar{\alpha}}$, which is the end of the edge $e_{\bar{\alpha}}$, has multiplicity equal to $m_{\bar{\alpha}}+1$. The $m_{\bar{\alpha}}$ edges, different from $e_{\bar{\alpha}}$, that branch out from $\mathcal{O}_{\bar{\alpha}}$ are denoted by $e \overline{\alpha \beta}$ with $\beta \in\left\{1, \ldots, m_{\bar{\alpha}}\right\}$. (See Figure 1.)

Let now $\mathcal{J}$ be the set of the interior vertices of $\Gamma$ and $\mathcal{E}$ be the set of the exterior vertices of $\Gamma, \mathcal{R}$ being excepted. We denote by

$$
I_{\mathcal{J}}=\left\{\bar{\alpha}, \mathcal{O}_{\bar{\alpha}} \in \mathcal{J}\right\}, \quad I_{\mathcal{E}}=\left\{\bar{\alpha}, \mathcal{O}_{\bar{\alpha}} \in \mathcal{E}\right\} .
$$

the sets of the indices for the interior and exterior vertices (except the root $\mathcal{R}$ ). With these notations $I=I_{\mathcal{J}} \cup I_{\mathcal{E}}$ is the set of the indices of all the vertices except the root $\mathcal{R}$.

The length of the edge $e_{\bar{\alpha}}$ will be denoted by $l_{\bar{\alpha}}$. Each $e_{\bar{\alpha}}$ is parameterized by the interval $\left[0, l_{\bar{\alpha}}\right]$, so that the end $\mathcal{O}_{\bar{\alpha}}$ of $e_{\bar{\alpha}}$ corresponds to $x=l_{\bar{\alpha}}$ while the origin of $e_{\bar{\alpha}}$ corresponds to $x=0$.

3.2. Carleman estimate for the heat equation. In this section we derive a Carleman estimate for the heat equation on a tree. The following properties for a function $\mathbf{u}=\left\{u^{\bar{\alpha}}\right\}_{\bar{\alpha} \in I}$ : $\Gamma \rightarrow \mathbb{R}$ will be relevant for our work.

(C1) Continuity condition at the internal vertices: $u^{\bar{\alpha}}\left(l_{\bar{\alpha}}\right)=u^{\overline{\alpha \beta}}(0)$ for all $\bar{\alpha} \in I_{\mathcal{J}}$ and $\beta \in$ $\left[\left[1, m_{\bar{\alpha}}\right]\right]$.

(C2) Flux condition at the internal vertices: $u_{x}^{\bar{\alpha}}\left(l_{\bar{\alpha}}\right)=\sum_{\beta=1}^{m_{\bar{\alpha}}} u_{x}^{\overline{\alpha \beta}}(0)$ for all $\bar{\alpha} \in I_{\mathcal{J}}$.

(C3) Vanishing condition at the root $\mathcal{R}$ and at the external vertices: $\mathbf{u}(v)=0$ for all $v \in\{\mathcal{R}\} \cup \mathcal{E}$.

We introduce the set

$$
\mathcal{Z}=\left\{\mathbf{u}=\left\{u^{\bar{\alpha}}\right\}_{\bar{\alpha} \in I}: \Gamma \times[0, T] \rightarrow \mathbb{R} ; u^{\bar{\alpha}} \in C^{2,1}\left(\left[0, l_{\bar{\alpha}}\right] \times[0, T]\right), \mathbf{u}(\cdot, t) \text { satisfies }(\mathrm{C} 1)-(\mathrm{C} 3)\right\} .
$$

Note that $\mathbf{u}(\cdot, t) \in D\left(\Delta_{\Gamma}\right)$ for $\mathbf{u} \in \mathcal{Z}$ and $t \in[0, T]$. The aim of this section is to define a continuous weight function $\boldsymbol{\psi}=\left\{\psi^{\bar{\alpha}}\right\}_{\bar{\alpha} \in I}: \Gamma \rightarrow(0, \infty)$ and a constant $C_{\boldsymbol{\psi}}>0$ such that if we 


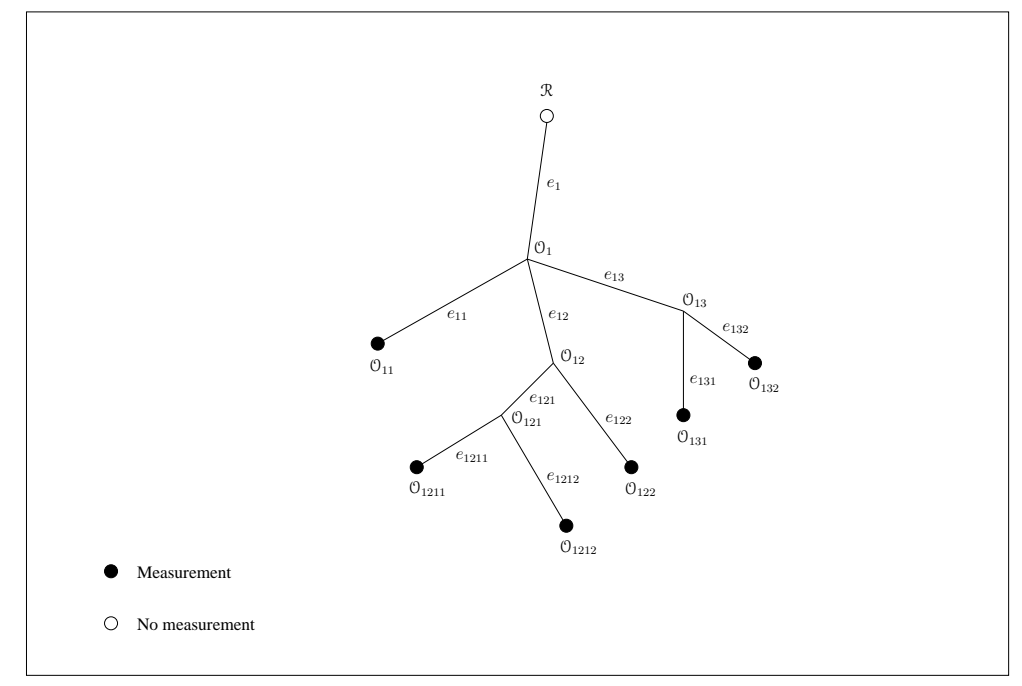

Figure 1. A tree with 10 edges.

set

$$
\boldsymbol{\theta}(x, t)=\frac{e^{\lambda \boldsymbol{\psi}(x)}}{t(T-t)}, \quad \boldsymbol{\varphi}(x, t)=\frac{e^{\lambda C_{\psi}}-e^{\lambda \boldsymbol{\psi}(x)}}{t(T-t)}, x \in \Gamma, t \in(0, T),
$$

we have the following Carleman estimate.

Proposition 3.1. There exist a continuous function $\psi: \Gamma \rightarrow(0,+\infty)$ and some positive constants $\lambda_{0}, s_{0}, C$ such that for all $\lambda \geq \lambda_{0}, s \geq s_{0}$ and $\mathbf{q} \in Z$, it holds

$$
\begin{aligned}
\int_{0}^{T} & \int_{\Gamma}\left((s \boldsymbol{\theta})^{-1}\left(\left|\mathbf{q}_{t}\right|^{2}+\left|\Delta_{\Gamma} \mathbf{q}\right|^{2}\right)+\lambda^{2}(s \boldsymbol{\theta})\left|\mathbf{q}_{x}\right|^{2}+\lambda^{4}(s \boldsymbol{\theta})^{3}|\mathbf{q}|^{2}\right) e^{-2 s \boldsymbol{\varphi}} d x d t \\
& +\int_{0}^{T} \lambda(s \boldsymbol{\theta})\left(\left|\mathbf{q}_{x}\right|^{2} e^{-2 s \boldsymbol{\varphi}}\right)(\mathcal{R}, t) d t \\
& \leq C\left(\int_{0}^{T} \int_{\Gamma}\left|\mathbf{q}_{t}+\Delta_{\Gamma} \mathbf{q}\right|^{2} e^{-2 s \boldsymbol{\varphi}} d x d t+\sum_{v \in \mathcal{E}} \int_{0}^{T} \lambda(s \boldsymbol{\theta})\left(\left|\mathbf{q}_{x}\right|^{2} e^{-2 s \boldsymbol{\varphi}}\right)(v, t) d t\right) .
\end{aligned}
$$

In the above proposition we have used the following notations $\left.\left|\mathbf{q}^{2}=\left\{\left|q^{\bar{\alpha}}\right|^{2}\right\}_{\bar{\alpha} \in I},\right| \mathbf{q}_{t}\right|^{2}=$ $\left\{\left|q_{t}^{\bar{\alpha}}\right|^{2}\right\}_{\bar{\alpha} \in I},\left|\mathbf{q}_{x}\right|^{2}=\left\{\left|q_{x}^{\bar{\alpha}}\right|^{2}\right\}_{\bar{\alpha} \in I}$, etc. and

$$
\int_{\Gamma} \mathbf{u} d x=\sum_{\bar{\alpha} \in I} \int_{I_{\bar{\alpha}}} u^{\bar{\alpha}} d x .
$$

Note that the same inequality holds for the operator $\partial_{t}-\Delta_{\Gamma}$ instead of $\partial_{t}+\Delta_{\Gamma}$ just by changing $t$ into $T-t$. Note also that in the definition of $z$ we can replace $C^{2,1}$ by $H^{2,1}$, as well.

Proof. Let us consider the operator $P=\partial_{t}+\Delta_{\Gamma}$. Set $\mathbf{u}=e^{-s \boldsymbol{\varphi}} \mathbf{q}$ and $\mathbf{w}=e^{-s \varphi} P\left(e^{s \varphi} \mathbf{u}\right)$. Following [32] we obtain

$$
\mathbf{w}=M \mathbf{u}=\mathbf{u}_{t}+s \varphi_{t} \mathbf{u}+\left(\Delta_{\Gamma} \mathbf{u}+2 s \varphi_{x} \mathbf{u}_{x}+s\left(\Delta_{\Gamma} \boldsymbol{\varphi}\right) \mathbf{u}+s^{2}\left|\varphi_{x}\right|^{2} \mathbf{u}\right)=M_{1} \mathbf{u}+M_{2} \mathbf{u}
$$


where

$$
M_{1} \mathbf{u}=\Delta_{\Gamma} \mathbf{u}+s \varphi_{t} \mathbf{u}+s^{2}\left|\varphi_{x}\right|^{2} \mathbf{u}
$$

and

$$
M_{2} \mathbf{u}=\mathbf{u}_{t}+2 s \varphi_{x} \mathbf{u}_{x}+s\left(\Delta_{\Gamma} \boldsymbol{\varphi}\right) \mathbf{u}
$$

are the self-adjoint and skew-adjoint parts of $M$, respectively. Then

$$
\|\mathbf{w}\|^{2}=\left\|M_{1} \mathbf{u}+M_{2} \mathbf{u}\right\|^{2}=\left\|M_{1} \mathbf{u}\right\|^{2}+\left\|M_{2} \mathbf{u}\right\|^{2}+2\left(M_{1} \mathbf{u}, M_{2} \mathbf{u}\right),
$$

where $\|\cdot\|$ and $(\cdot, \cdot)$ denote the norm and the inner product of $L^{2}(\Gamma \times(0, T))$, respectively.

Step 1. Exact computation of $\left(M_{1} \mathbf{u}, M_{2} \mathbf{u}\right)$.

Recall that

$$
\left(M_{1} \mathbf{u}, M_{2} \mathbf{u}\right)=\sum_{\bar{\alpha} \in I} \int_{0}^{T} \int_{0}^{l_{\bar{\alpha}}}\left(M_{1} \mathbf{u}\right)^{\bar{\alpha}}\left(M_{2} \mathbf{u}\right)^{\bar{\alpha}} d x d t .
$$

We compute the integral term in the r.h.s. of the above identity only for one (arbitrary) edge $e_{\bar{\alpha}}$, that we denote by $e$ for simplicity. We assume that $e$ is parameterized by $x \in[0, l]$. Also, where there is no confusion, we use the symbols $\iint$ and $\int$ to denote $\int_{0}^{T} \int_{0}^{l}$ and $\int_{0}^{T}$, respectively.

We write

$$
\int_{0}^{T} \int_{0}^{l}\left(M_{1} u\right)\left(M_{2} u\right) d x d t=I_{1}+I_{2}+I_{3}+I_{4}
$$

with

$$
\begin{aligned}
& I_{1}=\int_{0}^{T} \int_{0}^{l} u_{x x} u_{t}, \\
& I_{2}=\int_{0}^{T} \int_{0}^{l} u_{x x}\left(2 s \varphi_{x} u_{x}+s \varphi_{x x} u\right), \\
& I_{3}=\int_{0}^{T} \int_{0}^{l}\left(s \varphi_{t} u+s^{2} \varphi_{x}^{2} u\right)\left(u_{t}+2 s \varphi_{x} u_{x}\right), \\
& I_{4}=\int_{0}^{T} \int_{0}^{l}\left(s \varphi_{t} u+s^{2} \varphi_{x}^{2} u\right)\left(s \varphi_{x x} u\right) .
\end{aligned}
$$

For $I_{1}$ we have that

$$
I_{1}=-\iint u_{x} u_{x t}+\left.\int u_{x} u_{t}\right|_{0} ^{l}=\left.\int u_{x} u_{t}\right|_{0} ^{l} .
$$

We write the second term as $I_{2}=I_{2}^{1}+I_{2}^{2}$ where

$$
I_{2}^{1}=2 s \iint u_{x x} \varphi_{x} u_{x} \quad \text { and } \quad I_{2}^{2}=s \iint u_{x x} \varphi_{x x} u \text {. }
$$

Thus

$$
I_{2}^{1}=-s \iint \varphi_{x x}\left|u_{x}\right|^{2}+\left.s \int \varphi_{x}\left|u_{x}\right|^{2}\right|_{0} ^{l}
$$


and

$$
\begin{aligned}
I_{2}^{2} & =-s \iint u_{x}\left(\varphi_{3 x} u+\varphi_{x x} u_{x}\right)+\left.s \int u_{x} \varphi_{x x} u\right|_{0} ^{l} \\
& =\frac{s}{2} \iint \varphi_{4 x} u^{2}-\left.s \int \varphi_{3 x} \frac{u^{2}}{2}\right|_{0} ^{l}-s \iint \varphi_{x x}\left|u_{x}\right|^{2}+\left.s \int \varphi_{x x} u u_{x}\right|_{0} ^{l} .
\end{aligned}
$$

$I_{3}$ is decomposed as $I_{3}=I_{3}^{1}+I_{3}^{2}$ where

$$
\begin{array}{r}
I_{3}^{1}=\iint\left(s \varphi_{t} u+s^{2}\left|\varphi_{x}\right|^{2} u\right) u_{t} \\
I_{3}^{2}=\iint\left(s \varphi_{t} u+s^{2}\left|\varphi_{x}\right|^{2} u\right)\left(2 s \varphi_{x} u_{x}\right) .
\end{array}
$$

Then

$$
\begin{gathered}
I_{3}^{1}=-\iint\left(s \varphi_{t t}+2 s^{2} \varphi_{x} \varphi_{x t}\right) \frac{|u|^{2}}{2}, \\
I_{3}^{2}=\iint s \varphi_{x}\left(s \varphi_{t}+s^{2}\left|\varphi_{x}\right|^{2}\right) \partial_{x}\left(|u|^{2}\right) \\
=-s^{2} \iint\left(\left(\varphi_{x} \varphi_{t}\right)_{x}+s\left(\varphi_{x}^{3}\right)_{x}\right)|u|^{2}+\left.\int s \varphi_{x}\left(s \varphi_{t}+s^{2}\left|\varphi_{x}\right|^{2}\right)|u|^{2}\right|_{0} ^{l} .
\end{gathered}
$$

Finally,

$$
I_{4}=\iint\left(s^{2} \varphi_{t}+s^{3}\left|\varphi_{x}\right|^{2}\right) \varphi_{x x}|u|^{2} .
$$

We conclude that for the edge $e$,

$$
\begin{gathered}
\int_{0}^{T} \int_{0}^{l} M_{1} u M_{2} u d x d t=-2 s \int_{0}^{T} \int_{0}^{l} \varphi_{x x}\left|u_{x}\right|^{2}+\int_{0}^{T} \int_{0}^{l}|u|^{2}\left[\frac{s}{2}\left(\varphi_{4 x}-\varphi_{t t}\right)-s^{2}\left(\left|\varphi_{x}\right|^{2}\right)_{t}-s^{3} \varphi_{x}\left(\left|\varphi_{x}\right|^{2}\right)_{x}\right] \\
+\left.\int_{0}^{T}\left[u_{x} u_{t}+s \varphi_{x x} u u_{x}+s\left|u_{x}\right|^{2} \varphi_{x}+|u|^{2}\left(-\frac{s}{2} \varphi_{3 x}+s^{2} \varphi_{x} \varphi_{t}+s^{3}\left(\varphi_{x}\right)^{3}\right)\right]\right|_{0} ^{l}
\end{gathered}
$$

Summing now the above identity over all the edges $\left\{e_{\bar{\alpha}}\right\}_{\bar{\alpha} \in I}$ we obtain the exact expression of the scalar product $\left(M_{1} \mathbf{u}, M_{2} \mathbf{u}\right)$ :

$$
\begin{gathered}
\left(M_{1} \mathbf{u}, M_{2} \mathbf{u}\right)=-2 s \int_{0}^{T} \int_{\Gamma}\left(\Delta_{\Gamma} \boldsymbol{\varphi}\right)\left|\mathbf{u}_{x}\right|^{2}+\int_{0}^{T} \int_{\Gamma}|\mathbf{u}|^{2}\left[\frac{s}{2}\left(\boldsymbol{\varphi}_{4 x}-\boldsymbol{\varphi}_{t t}\right)-s^{2}\left(\left|\boldsymbol{\varphi}_{x}\right|^{2}\right)_{t}-s^{3} \boldsymbol{\varphi}_{x}\left(\left|\boldsymbol{\varphi}_{x}\right|^{2}\right)_{x}\right] \\
\quad+\left.\sum_{\bar{\alpha} \in I} \int_{0}^{T}\left[u_{x}^{\bar{\alpha}} u_{t}^{\bar{\alpha}}+s \varphi_{x x}^{\bar{\alpha}} u^{\bar{\alpha}} u_{x}^{\bar{\alpha}}+s\left|u_{x}^{\bar{\alpha}}\right|^{2} \varphi_{x}^{\bar{\alpha}}+\left|u^{\bar{\alpha}}\right|^{2}\left(-\frac{s}{2} \varphi_{3 x}^{\bar{\alpha}}+s^{2} \varphi_{x}^{\bar{\alpha}} \varphi_{t}^{\bar{\alpha}}+s^{3}\left(\varphi_{x}^{\bar{\alpha}}\right)^{3}\right)\right]\right|_{0} ^{l_{\bar{\alpha}}} \cdot(3.7)
\end{gathered}
$$

Step 2. Terms in the inner product related to the internal nodes.

Let us pick an internal node $\mathcal{O}_{\bar{\alpha}}$. Using our previous notations, its parent edge is $e_{\bar{\alpha}}$ and its children edges are denoted by $e_{\overline{\alpha \beta}}$ with $\beta \in\left[\left[1, m_{\bar{\alpha}}\right]\right]$. Let us denote by $X^{\bar{\alpha}}$ the sum of the 
boundary terms involving this internal node $\mathcal{O}_{\bar{\alpha}}$ in the right hand side of (3.7). Thus

$$
\begin{gathered}
X^{\bar{\alpha}}=\int_{0}^{T}\left[u_{x}^{\bar{\alpha}} u_{t}^{\bar{\alpha}}+s \varphi_{x x}^{\bar{\alpha}} u^{\bar{\alpha}} u_{x}^{\bar{\alpha}}+s\left|u_{x}^{\bar{\alpha}}\right|^{2} \varphi_{x}^{\bar{\alpha}}+\left|u^{\bar{\alpha}}\right|^{2}\left(-\frac{s}{2} \varphi_{3 x}^{\bar{\alpha}}+s^{2} \varphi_{x}^{\bar{\alpha}} \varphi_{t}^{\bar{\alpha}}+s^{3}\left(\varphi_{x}^{\bar{\alpha}}\right)^{3}\right)\right]\left(l_{\bar{\alpha}}, t\right) d t \\
-\int_{0}^{T} \sum_{\beta \in\left[\left[1, m_{\bar{\alpha}}\right]\right]}\left[u_{x}^{\overline{\alpha \beta}} u_{t}^{\overline{\alpha \beta}}+s \varphi_{x x}^{\overline{\alpha \beta}} u^{\overline{\alpha \beta}} u_{x}^{\overline{\alpha \beta}}+s\left|u_{x}^{\overline{\alpha \beta}}\right|^{2} \varphi_{x}^{\overline{\alpha \beta}}\right. \\
\left.+\left|u^{\overline{\alpha \beta}}\right|^{2}\left(-\frac{s}{2} \varphi_{3 x}^{\overline{\alpha \beta}}+s^{2} \varphi_{x}^{\overline{\alpha \beta}} \varphi_{t}^{\overline{\alpha \beta}}+s^{3}\left(\varphi_{x}^{\overline{\alpha \beta}}\right)^{3}\right)\right](0, t) d t .
\end{gathered}
$$

Moreover, in (3.7) we also have contributions from the exterior nodes in $\mathcal{E}$ and from the root $\mathcal{R}$. These contributions are given by

$$
Y=-s \int_{0}^{T}\left|u_{x}^{\overline{1}}\right|^{2} \varphi_{x}^{\overline{1}}(0, t) d t+s \sum_{\bar{\alpha} \in I_{\mathcal{E}}} \int_{0}^{T}\left|u_{x}^{\bar{\alpha}}\right|^{2} \varphi_{x}^{\bar{\alpha}}\left(l_{\bar{\alpha}}, t\right) d t .
$$

Let us now define the weight function $\boldsymbol{\psi}=\left\{\psi^{\bar{\alpha}}\right\}_{\bar{\alpha} \in I}$ on the tree as follows. The components $\psi^{\bar{\alpha}}:\left[0, l_{\bar{\alpha}}\right] \rightarrow \mathbb{R}$ are chosen in such a way that $\psi^{\bar{\alpha}} \in C^{\infty}\left(\left[0, l_{\bar{\alpha}}\right]\right)$ and

(B1) $\left|\psi_{x}^{\bar{\alpha}}(x)\right|^{2}+\psi_{x x}^{\bar{\alpha}}(x) \geq 0$ on $\left[0, l_{\bar{\alpha}}\right]$,

(B2) $\psi_{x}^{\bar{\alpha}}>0$ on $\left[0, l_{\bar{\alpha}}\right]$,

(B3) $\frac{3}{4} C_{\boldsymbol{\psi}} \geq \psi^{\bar{\alpha}}>\frac{2}{3} C_{\boldsymbol{\psi}}$ on $\left[0, l_{\bar{\alpha}}\right]$, for some positive constant $C_{\boldsymbol{\psi}}$,

(B4) $\left|\psi_{x x}^{\bar{\alpha}}\right| \leq K \psi_{x}^{\bar{\alpha}}$ on $\left[0, l_{\bar{\alpha}}\right]$ for some positive constant $K$,

(B5) $\psi^{\bar{\alpha}}\left(l_{\bar{\alpha}}\right)=\psi^{\overline{\alpha \beta}}(0)$ for all $\bar{\alpha} \in I_{\mathcal{J}}, \beta \in\left[\left[1, m_{\bar{\alpha}}\right]\right]$,

(B6) $\psi_{x}^{\overline{\alpha \beta}}(0)-\left(m_{\bar{\alpha}}+1\right) \psi_{x}^{\bar{\alpha}}\left(l_{\bar{\alpha}}\right)>0$ for all $\bar{\alpha} \in I_{\mathcal{J}}, \beta \in\left[\left[1, m_{\bar{\alpha}}\right]\right]$,

(B7) $\sum_{\beta \in\left[\left[1, m_{\bar{\alpha}}\right]\right]}\left(\psi_{x}^{\overline{\alpha \beta}}(0)\right)^{3}-\left(\psi_{x}^{\bar{\alpha}}\left(l_{\bar{\alpha}}\right)\right)^{3}-\left(m_{\bar{\alpha}}+1\right) \psi_{x}^{\bar{\alpha}}\left(l_{\bar{\alpha}}\right)\left|\psi_{x}^{\bar{\alpha}}\left(l_{\bar{\alpha}}\right)-\sum_{\beta \in\left[\left[1, m_{\bar{\alpha}}\right]\right]} \psi_{x}^{\overline{\alpha \beta}}(0)\right|^{2}>0$ for all $\bar{\alpha} \in I_{\mathcal{J}}$.

Finding a set of functions as above is easy. We can even take $\psi^{\bar{\alpha}}$ to be affine, $\psi^{\bar{\alpha}}(x)=a_{\bar{\alpha}} x+b_{\bar{\alpha}}$. The coefficients $a_{\bar{\alpha}}$ and $b_{\bar{\alpha}}$ are positive numbers that satisfy

(P1) $\frac{3}{4} C_{\psi} \geq a_{\bar{\alpha}} l_{\bar{\alpha}}+b_{\bar{\alpha}}>b_{\bar{\alpha}}>\frac{2}{3} C_{\psi}$ for all $\bar{\alpha} \in I$,

(P2) $a_{\bar{\alpha}} l_{\bar{\alpha}}+b_{\bar{\alpha}}=b \overline{\alpha \beta}$ for all $\bar{\alpha} \in I_{\mathcal{J}}$ and $\beta \in\left[\left[1, m_{\bar{\alpha}}\right]\right]$,

(P3) $a_{\overline{\alpha \beta}}-\left(m_{\bar{\alpha}}+1\right) a_{\bar{\alpha}}>0$ for all $\bar{\alpha} \in I_{\mathcal{J}}$ and $\beta \in\left[\left[1, m_{\bar{\alpha}}\right]\right]$,

(P4) $\sum_{\beta \in\left[\left[1, m_{\bar{\alpha}}\right]\right]}\left(a_{\overline{\alpha \beta}}\right)^{3}-\left(a_{\bar{\alpha}}\right)^{3}-\left(m_{\bar{\alpha}}+1\right) a_{\bar{\alpha}}\left|a_{\bar{\alpha}}-\sum_{\beta \in\left[\left[1, m_{\bar{\alpha}}\right]\right]} a_{\overline{\alpha \beta}}\right|^{2}>0$ for all $\bar{\alpha} \in I_{\mathcal{J}}$.

Let us first deal with the conditions (P2)-(P4). We define the constants corresponding to the edge $e_{\overline{1}}$ by $a_{\overline{1}}=2$ and $b_{\overline{1}}=1$. Assuming that we have already constructed $a_{\bar{\alpha}}$ and $b_{\bar{\alpha}}$ for some 
multi-index $\bar{\alpha}$, then $b_{\overline{\alpha \beta}}$ is given by (P2). Next, we have to find $a_{\overline{\alpha \beta}}$ large enough to satisfy (P3)(P4). Let us choose $a_{\overline{\alpha \beta}}=r_{\bar{\alpha}} a_{\bar{\alpha}}$. Obviously, for large enough $r_{\bar{\alpha}}$, depending on $m_{\bar{\alpha}}$, conditions (P3) and (P4) are satisfied. Finally, assume that all the coefficients $a_{\bar{\alpha}}$ and $b_{\bar{\alpha}}$ have been defined to satisfy (P2)-(P4). Adding $\frac{2}{3} C_{\boldsymbol{\psi}}$ to all the $b_{\overline{\alpha \beta}}$, we see that (P1) is fulfilled for $C_{\boldsymbol{\psi}}$ large enough, while (P2)-(P4) still hold true.

Let us split $X^{\bar{\alpha}}$ into $X^{\bar{\alpha}}=X_{1}^{\bar{\alpha}}+X_{2}^{\bar{\alpha}}+X_{3}^{\bar{\alpha}}+X_{4}^{\bar{\alpha}}$, where

$$
\begin{aligned}
& X_{1}^{\bar{\alpha}}:=\int_{0}^{T}\left[\left[u_{x}^{\bar{\alpha}} u_{t}^{\bar{\alpha}}\right]\left(l_{\bar{\alpha}}, t\right)-\sum_{\beta \in\left[\left[1, m_{\bar{\alpha}}\right]\right]}\left[u_{x}^{\overline{\alpha \beta}} u_{t}^{\overline{\alpha \beta}}\right](0, t)\right] d t \\
& X_{2}^{\bar{\alpha}}:=\int_{0}^{T}\left[\left[s \varphi_{x x}^{\bar{\alpha}} u^{\bar{\alpha}} u_{x}^{\bar{\alpha}}\right]\left(l_{\bar{\alpha}}, t\right)-\sum_{\beta \in\left[\left[1, m_{\bar{\alpha}}\right]\right]}\left[s \varphi_{x x}^{\overline{\alpha \beta}} u^{\overline{\alpha \beta}} u_{x}^{\overline{\alpha \beta}}\right](0, t)\right] d t, \\
& X_{3}^{\bar{\alpha}}:=\int_{0}^{T}\left[\left[s\left|u_{x}^{\bar{\alpha}}\right|^{2} \varphi_{x}^{\bar{\alpha}}\right]\left(l_{\bar{\alpha}}, t\right)-\sum_{\beta \in\left[\left[1, m_{\bar{\alpha}}\right]\right]}\left[s\left|u_{x}^{\overline{\alpha \beta}}\right|^{2} \varphi_{x}^{\overline{\alpha \beta}}\right](0, t)\right] d t \\
& X_{4}^{\bar{\alpha}}:=\int_{0}^{T}\left\{\left[\left|u^{\bar{\alpha}}\right|^{2}\left(-\frac{s}{2} \varphi_{3 x}^{\bar{\alpha}}+s^{2} \varphi_{x}^{\bar{\alpha}} \varphi_{t}^{\bar{\alpha}}+s^{3}\left(\varphi_{x}^{\bar{\alpha}}\right)^{3}\right)\right]\left(l_{\bar{\alpha}}, t\right)\right. \\
&\left.\quad \sum_{\beta \in\left[\left[1, m_{\bar{\alpha}}\right]\right]}\left[\left|u^{\overline{\alpha \beta}}\right|^{2}\left(-\frac{s}{2} \varphi_{3 x}^{\overline{\alpha \beta}}+s^{2} \varphi_{x}^{\overline{\alpha \beta}} \varphi_{t}^{\overline{\alpha \beta}}+s^{3}\left(\varphi_{x}^{\overline{\alpha \beta}}\right)^{3}\right)\right](0, t)\right\} d t .
\end{aligned}
$$

We now estimate each term $X_{i}^{\bar{\alpha}}, i=1, \ldots, 4$. Using the definition of the function $\mathbf{u}$ we have for any index $\bar{\alpha} \in I$ the following identities

$$
u_{x}^{\bar{\alpha}}=e^{-s \varphi^{\bar{\alpha}}}\left(-s \varphi_{x}^{\bar{\alpha}} q^{\bar{\alpha}}+q_{x}^{\bar{\alpha}}\right), \quad u_{t}^{\bar{\alpha}}=e^{-s \varphi^{\bar{\alpha}}}\left(-s \varphi_{t}^{\bar{\alpha}} q^{\bar{\alpha}}+q_{t}^{\bar{\alpha}}\right) .
$$

Let us set $u\left(\mathcal{O}_{\bar{\alpha}}, t\right)=u^{\bar{\alpha}}\left(l_{\bar{\alpha}}, t\right)=u^{\overline{\alpha \beta}}(0, t)$ and $\varphi\left(\mathcal{O}_{\bar{\alpha}}, t\right)=\varphi^{\bar{\alpha}}\left(l_{\bar{\alpha}}, t\right)=\varphi^{\overline{\alpha \beta}}(0, t)$ for any $\bar{\alpha} \in I_{\mathcal{J}}$ and $\beta \in\left[\left[1, m_{\bar{\alpha}}\right]\right]$. With these notations we have

$$
\begin{aligned}
X_{1}^{\bar{\alpha}} & =\int_{0}^{T}\left(u_{x}^{\bar{\alpha}} u_{t}^{\bar{\alpha}}\left(l_{\bar{\alpha}}, t\right)-\sum_{\beta \in\left[\left[1, m_{\bar{\alpha}}\right]\right]} u_{x}^{\overline{\alpha \beta}} u_{t}^{\overline{\alpha \beta}}(0, t)\right) d t \\
& =\int_{0}^{T} u_{t}\left(\mathcal{O}_{\bar{\alpha}}, t\right) e^{-s \varphi\left(\mathcal{O}_{\bar{\alpha}}, t\right)}\left(-s \varphi_{x}^{\bar{\alpha}} q^{\bar{\alpha}}\left(l_{\bar{\alpha}}\right)+s \sum_{\beta \in\left[\left[1, m_{\bar{\alpha}]}\right]\right]} \varphi_{x}^{\overline{\alpha \beta}} q^{\overline{\alpha \beta}}(0)+q_{x}^{\bar{\alpha}}\left(l_{\bar{\alpha}}\right)-\sum_{\beta \in\left[\left[1, m_{\bar{\alpha}]}\right]\right]} q_{x}^{\overline{\alpha \beta}}(0)\right) d t \\
& =\int_{0}^{T} u_{t}\left(\mathcal{O}_{\bar{\alpha}}, t\right) e^{-s \varphi\left(\mathcal{O}_{\bar{\alpha}}, t\right)}\left(-s \varphi_{x}^{\bar{\alpha}} q^{\bar{\alpha}}\left(l_{\bar{\alpha}}\right)+s \sum_{\beta \in\left[\left[1, m_{\bar{\alpha}}\right]\right]} \varphi_{x}^{\overline{\alpha \beta}} q^{\overline{\alpha \beta}}(0)\right) d t \\
& =\int_{0}^{T} u_{t}\left(\mathcal{O}_{\bar{\alpha}}, t\right) u\left(\mathcal{O}_{\bar{\alpha}}, t\right)\left(-s \varphi_{x}^{\bar{\alpha}}\left(l_{\bar{\alpha}}\right)+s \sum_{\beta \in\left[\left[1, m_{\bar{\alpha}}\right]\right]} \varphi_{x}^{\overline{\alpha \beta}}(0)\right) d t \\
& =-\int_{0}^{T}\left(-s \varphi_{x t}^{\bar{\alpha}}\left(l_{\bar{\alpha}}, t\right)+s \sum_{\beta \in\left[\left[1, m_{\bar{\alpha}]}\right]\right]} \varphi_{x t}^{\overline{\alpha \beta}}(0, t)\right) \frac{\left|u\left(\mathcal{O}_{\bar{\alpha}}, t\right)\right|^{2}}{2} d t .
\end{aligned}
$$


Let us estimate $X_{2}^{\bar{\alpha}}$. Using property (B4) we infer that

$$
\left|\varphi_{x x}^{\bar{\alpha}}\right|=\frac{\lambda e^{\lambda \psi^{\bar{\alpha}}}}{t(T-t)}\left|\lambda\left(\psi_{x}^{\bar{\alpha}}\right)^{2}+\psi_{x x}^{\bar{\alpha}}\right| \leq \frac{T^{2}}{4}\left|\varphi_{x}^{\bar{\alpha}}\right|^{2}+K\left|\varphi_{x}^{\bar{\alpha}}\right|
$$

This gives that

$$
\begin{aligned}
\left|X_{2}^{\bar{\alpha}}\right|= & \left|\int_{0}^{T}\left(s \varphi_{x x}^{\bar{\alpha}} u^{\bar{\alpha}} u_{x}^{\bar{\alpha}}\left(l_{\bar{\alpha}}, t\right)-s \sum_{\beta \in\left[\left[1, m_{\bar{\alpha}}\right]\right]} \varphi_{x x}^{\overline{\alpha \beta}} u^{\overline{\alpha \beta}} u_{x}^{\overline{\alpha \beta}}(0, t)\right) d t\right| \\
\leq & \frac{s^{2}}{2} \int_{0}^{T}\left|u^{\bar{\alpha}}\right|^{2}\left(\frac{T^{2}}{4}\left|\varphi_{x}^{\bar{\alpha}}\right|+K\right)^{2}\left|\varphi_{x}^{\bar{\alpha}}\right|\left(l_{\bar{\alpha}}, t\right) d t+\frac{1}{2} \int_{0}^{T}\left|\varphi_{x}^{\bar{\alpha}}\right|\left|u_{x}^{\bar{\alpha}}\right|^{2}\left(l_{\bar{\alpha}}, t\right) d t \\
& +\sum_{\beta \in\left[\left[1, m_{\bar{\alpha}]}\right]\right.}\left\{\frac{s^{2}}{2} \int_{0}^{T}\left|u^{\overline{\alpha \beta}}\right|^{2}\left(\frac{T^{2}}{4}\left|\varphi_{x}^{\overline{\alpha \beta}}\right|+K\right)^{2}\left|\varphi_{x}^{\overline{\alpha \beta}}\right|(0, t) d t+\frac{1}{2} \int_{0}^{T}\left|\varphi_{x}^{\overline{\alpha \beta}}\right|\left|u_{x}^{\overline{\alpha \beta}}\right|^{2}(0, t) d t\right\} \\
= & \frac{s^{2}}{2} \int_{0}^{T}\left|u\left(\mathcal{O}_{\bar{\alpha}}, t\right)\right|^{2}\left[\left(\frac{T^{2}}{4}\left|\varphi_{x}^{\bar{\alpha}}\right|+K\right)^{2}\left|\varphi_{x}^{\bar{\alpha}}\right|\left(l_{\bar{\alpha}}, t\right)+\sum_{\beta \in\left[\left[1, m_{\bar{\alpha}}\right]\right]}\left(\frac{T^{2}}{4}\left|\varphi_{x}^{\overline{\alpha \beta}}\right|+K\right)^{2}\left|\varphi_{x}^{\overline{\alpha \beta}}\right|(0, t)\right] d t \\
& +\frac{1}{2} \int_{0}^{T}\left[\left|\varphi_{x}^{\bar{\alpha}}\right|\left|u_{x}^{\bar{\alpha}}\right|^{2}\left(l_{\bar{\alpha}}, t\right) d t+\sum_{\beta \in\left[\left[1, m_{\bar{\alpha}}\right]\right]}\left|\varphi_{x}^{\overline{\alpha \beta}}\right|\left|u_{x}^{\overline{\alpha \beta}}\right|^{2}(0, t)\right] d t .
\end{aligned}
$$

To estimate the term $\int_{0}^{T}\left|\varphi_{x}^{\bar{\alpha}}\right|\left|u_{x}^{\bar{\alpha}}\right|^{2}\left(l_{\bar{\alpha}}, t\right) d t$ which occurs in (3.10) and in $X_{3}^{\bar{\alpha}}$, we notice that

$$
u_{x}^{\bar{\alpha}}\left(l_{\bar{\alpha}}, t\right)-\sum_{\beta \in\left[\left[1, m_{\bar{\alpha}}\right]\right]} u_{x}^{\overline{\alpha \beta}}(0, t)=e^{-s \varphi\left(\mathcal{O}_{\bar{\alpha}}, t\right)}(-s)\left(\varphi_{x}^{\bar{\alpha}}\left(l_{\bar{\alpha}}, t\right)-\sum_{\beta \in\left[\left[1, m_{\bar{\alpha}}\right]\right]} \varphi_{x}^{\overline{\alpha \beta}}(0, t)\right) q\left(\mathcal{O}_{\bar{\alpha}}, t\right)
$$

hence

$$
\left|u_{x}^{\bar{\alpha}}\left(l_{\bar{\alpha}}, t\right)\right|^{2} \leq\left(m_{\bar{\alpha}}+1\right)\left(\sum_{\beta \in\left[\left[1, m_{\bar{\alpha}}\right]\right]}\left|u_{x}^{\overline{\alpha \beta}}(0, t)\right|^{2}+s^{2}\left|u\left(\mathcal{O}_{\bar{\alpha}}, t\right)\right|^{2}\left|\varphi_{x}^{\bar{\alpha}}\left(l_{\bar{\alpha}}, t\right)-\sum_{\beta \in\left[\left[1, m_{\bar{\alpha}}\right]\right]} \varphi_{x}^{\overline{\alpha \beta}}(0, t)\right|^{2}\right) .
$$

We infer that

$$
\begin{aligned}
& \int_{0}^{T}\left|\varphi_{x}^{\bar{\alpha}}\right|\left|u_{x}^{\bar{\alpha}}\right|^{2}\left(l_{\bar{\alpha}}, t\right) d t \\
& \quad \leq\left(m_{\bar{\alpha}}+1\right) \int_{0}^{T}\left|\varphi_{x}^{\bar{\alpha}}\left(l_{\bar{\alpha}}, t\right)\right|\left(\sum_{\left.\beta \in\left[1, m_{\bar{\alpha}}\right]\right]}\left|u_{x}^{\overline{\alpha \beta}}(0, t)\right|^{2}+s^{2}\left|u\left(\mathcal{O}_{\bar{\alpha}}, t\right)\right|^{2}\left|\varphi_{x}^{\bar{\alpha}}\left(l_{\bar{\alpha}}, t\right)-\sum_{\beta \in\left[\left[1, m_{\bar{\alpha}}\right]\right]} \varphi_{x}^{\overline{\alpha \beta}}(0, t)\right|^{2}\right)
\end{aligned}
$$


Combined to (3.10) and to the fact that $\varphi_{x}^{\overline{\alpha \beta}}(0, t) \leq 0$ by $(\mathrm{B} 2)$, this yields

$$
\begin{aligned}
& X_{2}^{\bar{\alpha}}+X_{3}^{\bar{\alpha}} \\
& \geq-\frac{s^{2}}{2} \int_{0}^{T}\left|u\left(\mathcal{O}_{\bar{\alpha}}, t\right)\right|^{2}\left[\left(\frac{T^{2}}{4}\left|\varphi_{x}^{\bar{\alpha}}\right|+K\right)^{2}\left|\varphi_{x}^{\bar{\alpha}}\right|\left(l_{\bar{\alpha}}, t\right)+\sum_{\beta \in\left[\left[1, m_{\bar{\alpha}}\right]\right]}\left(\frac{T^{2}}{4}\left|\varphi_{x}^{\overline{\alpha \beta}}\right|+K\right)^{2}\left|\varphi_{x}^{\overline{\alpha \beta}}\right|(0, t)\right] d t \\
& +\left(s-\frac{1}{2}\right) \int_{0}^{T} \sum_{\beta \in\left[\left[1, m_{\bar{\alpha}]}\right]\right]}\left|\varphi_{x}^{\overline{\alpha \beta}}\right|\left|u_{x}^{\overline{\alpha \beta}}\right|^{2}(0, t) d t \\
& -\left(s+\frac{1}{2}\right)\left(m_{\bar{\alpha}}+1\right) \int_{0}^{T}\left|\varphi_{x}^{\bar{\alpha}}\left(l_{\bar{\alpha}}, t\right)\right|\left(\sum_{\beta \in\left[\left[1, m_{\bar{\alpha}}\right]\right]}\left|u_{x}^{\overline{\alpha \beta}}(0, t)\right|^{2}+s^{2}\left|u\left(\mathcal{O}_{\bar{\alpha}}, t\right)\right|^{2}\left|\varphi_{x}^{\bar{\alpha}}\left(l_{\bar{\alpha}}, t\right)-\sum_{\beta \in\left[\left[1, m_{\bar{\alpha}}\right]\right]} \varphi_{x}^{\overline{\alpha \beta}}(0, t)\right|^{2}\right) .
\end{aligned}
$$

Using the definition of $X^{\bar{\alpha}}$ and estimates (3.9), (3.13) we obtain that

$$
X^{\bar{\alpha}} \geq Z_{1}^{\bar{\alpha}}+Z_{2}^{\bar{\alpha}}
$$

where

$$
\begin{aligned}
Z_{1}^{\bar{\alpha}} & =-\int_{0}^{T}\left(-s \varphi_{x t}^{\bar{\alpha}}\left(l_{\bar{\alpha}}, t\right)+s \sum_{\beta \in\left[\left[1, m_{\bar{\alpha}}\right]\right]} \varphi_{x t}^{\overline{\alpha \beta}}(0, t)\right) \frac{\left|u\left(\mathcal{O}_{\bar{\alpha}}, t\right)\right|^{2}}{2} d t \\
& -\frac{s^{2}}{2} \int_{0}^{T}\left|u\left(\mathcal{O}_{\bar{\alpha}}, t\right)\right|^{2}\left[\left(\frac{T^{2}}{4}\left|\varphi_{x}^{\bar{\alpha}}\right|+K\right)^{2}\left|\varphi_{x}^{\bar{\alpha}}\right|\left(l_{\bar{\alpha}}, t\right)+\sum_{\beta \in\left[\left[1, m_{\bar{\alpha}}\right]\right]}\left(\frac{T^{2}}{4}\left|\varphi_{x}^{\overline{\alpha \beta}}\right|+K\right)^{2}\left|\varphi_{x}^{\overline{\alpha \beta}}\right|(0, t)\right] d t \\
& -\left(s^{3}+\frac{1}{2} s^{2}\right)\left(m_{\bar{\alpha}}+1\right) \int_{0}^{T}\left|u\left(\mathcal{O}_{\bar{\alpha}}, t\right)\right|^{2}\left|\varphi_{x}^{\bar{\alpha}}\left(l_{\bar{\alpha}}, t\right)\right|\left|\varphi_{x}^{\bar{\alpha}}\left(l_{\bar{\alpha}}, t\right)-\sum_{\beta \in\left[\left[1, m_{\bar{\alpha}}\right]\right]} \varphi_{x}^{\overline{\alpha \beta}}(0, t)\right|^{2} d t \\
& \left.+\int_{0}^{T}\left|u\left(\mathcal{O}_{\bar{\alpha}}, t\right)\right|^{2} \mid\left(-\frac{s}{2} \varphi_{3 x}^{\bar{\alpha}}+s^{2} \varphi_{x}^{\bar{\alpha}} \varphi_{t}^{\bar{\alpha}}+s^{3}\left(\varphi_{x}^{\bar{\alpha}}\right)^{3}\right)\right]\left(l_{\bar{\alpha}}, t\right) d t \\
& -\int_{0}^{T}\left|u\left(\mathcal{O}_{\bar{\alpha}}, t\right)\right|^{2} \sum_{\beta \in\left[\left[1, m_{\bar{\alpha}}\right]\right]}\left(-\frac{s}{2} \varphi_{3 x}^{\overline{\alpha \beta}}+s^{2} \varphi_{x}^{\overline{\alpha \beta}} \varphi_{t}^{\overline{\alpha \beta}}+s^{3}\left(\varphi_{x}^{\overline{\alpha \beta}}\right)^{3}\right)(0, t) d t
\end{aligned}
$$

and

$$
Z_{2}^{\bar{\alpha}}=\left(s-\frac{1}{2}\right) \sum_{\beta \in\left[\left[1, m_{\bar{\alpha}}\right]\right]} \int_{0}^{T}\left[\left|\varphi_{x}^{\overline{\alpha \beta}}\right|\left|u_{x}^{\overline{\alpha \beta}}\right|^{2}\right](0, t) d t-\left(s+\frac{1}{2}\right)\left(m_{\bar{\alpha}}+1\right) \int_{0}^{T}\left[\left|\varphi_{x}^{\bar{\alpha}}\left(l_{\bar{\alpha}}, t\right)\right| \sum_{\beta \in\left[\left[1, m_{\bar{\alpha}}\right]\right]}\left|u_{x}^{\overline{\alpha \beta}}(0, t)\right|^{2}\right] d t .
$$

We notice that $\left|\varphi_{x}^{\bar{\alpha}}\right|^{3} \geq c(\lambda \theta)^{3}$ while, with (B3),

$$
\left|\varphi_{x}^{\bar{\alpha}}\right|+\left|\varphi_{x}^{\bar{\alpha}}\right|^{2}+\left|\varphi_{x t}^{\bar{\alpha}}\right|+\left|\varphi_{x}^{\bar{\alpha}} \varphi_{t}^{\bar{\alpha}}\right|+\left|\varphi_{3 x}^{\bar{\alpha}}\right| \leq c(\lambda \theta)^{3} .
$$


It follows that

$$
\begin{aligned}
Z_{1}^{\bar{\alpha}} & =\int_{0}^{T}\left|u\left(\mathcal{O}_{\bar{\alpha}}, t\right)\right|^{2} \times \\
& \times\left[s^{3}\left(\left(\varphi_{x}^{\bar{\alpha}}\right)^{3}\left(l_{\bar{\alpha}}, t\right)-\sum_{\beta \in\left[\left[1, m_{\bar{\alpha}}\right]\right]}\left(\varphi_{x}^{\overline{\alpha \beta}}\right)^{3}(0, t)-\left(m_{\bar{\alpha}}+1\right)\left|\varphi_{x}^{\bar{\alpha}}\left(l_{\bar{\alpha}}, t\right)\right|\left|\varphi_{x}^{\bar{\alpha}}\left(l_{\bar{\alpha}}, t\right)-\sum_{\beta \in\left[\left[1, m_{\bar{\alpha}]}\right]\right.} \varphi_{x}^{\overline{\alpha \beta}}(0, t)\right|^{2}\right)\right. \\
& +\ldots] \\
& =\int_{0}^{T}\left|u\left(\mathcal{O}_{\bar{\alpha}}, t\right)\right|^{2} \times \\
& \times\left[(s \lambda \theta)^{3}\left(-\left(\psi_{x}^{\bar{\alpha}}\right)^{3}\left(l_{\bar{\alpha}}\right)+\sum_{\beta \in\left[\left[1, m_{\bar{\alpha}}\right]\right]}\left(\psi_{x}^{\overline{\alpha \beta}}\right)^{3}(0)-\left(m_{\bar{\alpha}}+1\right)\left|\psi_{x}^{\bar{\alpha}}\left(l_{\bar{\alpha}}\right)\right|\left|\psi_{x}^{\bar{\alpha}}\left(l_{\bar{\alpha}}\right)-\sum_{\beta \in\left[\left[1, m_{\bar{\alpha}}\right]\right]} \psi_{x}^{\overline{\alpha \beta}}(0)\right|^{2}\right)\right. \\
& \left.+O\left(s^{2} \lambda^{3} \theta^{3}\right)\right]
\end{aligned}
$$

and

$$
Z_{2}^{\bar{\alpha}}=\int_{0}^{T} \sum_{\beta \in\left[\left[1, m_{\bar{\alpha}}\right]\right]}\left|u_{x}^{\overline{\alpha \beta}}(0, t)\right|^{2}\left[s \lambda \theta\left(\psi_{x}^{\overline{\alpha \beta}}(0)-\left(m_{\bar{\alpha}}+1\right)\left|\psi_{x}^{\bar{\alpha}}\left(l_{\bar{\alpha}}\right)\right|\right)+O(\lambda \theta)\right] d t .
$$

Looking at the coefficient of $s^{3}$ in $Z_{1}^{\bar{\alpha}}$ and of $s$ in $Z_{2}^{\bar{\alpha}}$ and using (B6), (B7), and (3.12), we obtain that for $s \geq s_{0}$ and $\lambda \geq \lambda_{0}$ (with $s_{0}, \lambda_{0}$ large enough)

$$
\begin{aligned}
X^{\bar{\alpha}} & \geq Z_{1}^{\bar{\alpha}}+Z_{2}^{\bar{\alpha}} \\
& \geq C \int_{0}^{T} s^{3} \lambda^{3} \theta^{3}\left|u\left(\mathcal{O}_{\bar{\alpha}}, t\right)\right|^{2} d t+C \int_{0}^{T} s \lambda \theta\left(\left|u_{x}^{\bar{\alpha}}\left(l_{\bar{\alpha}}, t\right)\right|^{2}+\sum_{\left.\beta \in\left[1, m_{\bar{\alpha}}\right]\right]}\left|u_{x}^{\overline{\alpha \beta}}(0, t)\right|^{2}\right) d t
\end{aligned}
$$

In particular, $X^{\bar{\alpha}}>0$.

Step 3. Estimation of the integrals along the edges.

We need the following lemma.

Lemma 3.2. [32, Claim 1] There exist $\lambda_{1} \geq \lambda_{0}, s_{1} \geq s_{0}$ and $A>0$ such that for all $\lambda \geq \lambda_{1}$, $s \geq s_{1}$, it holds

$$
\int_{0}^{T} \int_{\Gamma}|\mathbf{u}|^{2}\left[\frac{s}{2}\left(\varphi_{4 x}-\varphi_{t t}\right)-s^{2}\left(\left|\varphi_{x}\right|^{2}\right)_{t}-s^{3} \varphi_{x}\left(\left|\varphi_{x}\right|^{2}\right)_{x}\right] d x d t \geq A \lambda s^{3} \int_{0}^{T} \int_{\Gamma}|\mathbf{u}|^{2}\left|\varphi_{x}\right|^{3} d x d t .
$$

As the proof of [32, Claim 1] does not involve any integration by parts in $x$, it is still valid in our context.

The following lemma is inspired by [32, Claim 2]. 
Lemma 3.3. There exist $s_{2} \geq s_{1}, \lambda_{2} \geq \lambda_{1}$, and a positive constant $C$ such that for all $\lambda \geq \lambda_{2}$ and $s \geq s_{2}$

$$
\begin{aligned}
\lambda s & \int_{0}^{T} \int_{\Gamma}\left|\varphi_{x}\right|\left|\mathbf{u}_{x}\right|^{2}+\lambda s^{-1} \int_{0}^{T} \int_{\Gamma}\left|\varphi_{x}\right|^{-1}\left|\Delta_{\Gamma} \mathbf{u}\right|^{2} \\
\leq & C\left(s^{-1}\left\|M_{1} \mathbf{u}\right\|^{2}+\lambda s^{3} \int_{0}^{T} \int_{\Gamma}\left|\varphi_{x}\right|^{3}|\mathbf{u}|^{2}+\lambda s \int_{0}^{T} \sum_{\bar{\alpha} \in I}\left|\varphi_{x}^{\bar{\alpha}} u^{\bar{\alpha}} u_{x}^{\bar{\alpha}}\right|(0)+\left|\varphi_{x}^{\bar{\alpha}} u^{\bar{\alpha}} u_{x}^{\bar{\alpha}}\right|\left(l_{\bar{\alpha}}\right)\right. \\
& \left.+\lambda s \int_{0}^{T} \sum_{\bar{\alpha} \in I}\left|\left(\left|\varphi_{x}^{\bar{\alpha}}\right|\right)_{x}\right|\left|u^{\bar{\alpha}}\right|^{2}(0)+\left|\left(\left|\varphi_{x}^{\bar{\alpha}}\right|\right)_{x}\right|\left|u^{\bar{\alpha}}\right|^{2}\left(l_{\bar{\alpha}}\right)\right) .
\end{aligned}
$$

Proof. Let us pick any edge $e_{\bar{\alpha}}$. To simplify the writing, we remove the index $\bar{\alpha}$ in our computations. Using the definition of $M_{1} \mathbf{u}$ (see (3.4)) we have that

$$
\begin{array}{r}
s^{-1} \int_{0}^{T} \int_{0}^{l}\left|\varphi_{x}\right|^{-1}\left|u_{x x}\right|^{2}=s^{-1} \int_{0}^{T} \int_{0}^{l}\left|\varphi_{x}\right|^{-1}\left|M_{1} u-s \varphi_{t} u-s^{2} \varphi_{x}^{2} u\right|^{2} \\
\leq C s^{-1} \int_{0}^{T} \int_{0}^{l}\left|\varphi_{x}\right|^{-1}\left[\left|M_{1} u\right|^{2}+s^{2}\left|\varphi_{t}\right|^{2} u^{2}+s^{4}\left|\varphi_{x}\right|^{4} u^{2}\right] .
\end{array}
$$

Using property (B3) we get that $\left|\varphi_{t}\right| \leq C\left|\varphi_{x}\right|^{2}$. Therefore, we have for some constant $A>0$

$$
s^{-1} \int_{0}^{T} \int_{0}^{l}\left|\varphi_{x}\right|^{-1}\left|u_{x x}\right|^{2} \leq A\left(\frac{\left\|M_{1} u\right\|^{2}}{\lambda s}+s^{3} \int_{0}^{T} \int_{0}^{l}\left|\varphi_{x}\right|^{3} u^{2}\right) .
$$

The first term in the left hand side of (3.16) satisfies

$$
\begin{aligned}
& \lambda s \iint\left|\varphi_{x}\right|\left|u_{x}\right|^{2}=\lambda s\left(\iint\left|\varphi_{x}\right|\left(-u_{x x}\right) u-\iint\left(\left|\varphi_{x}\right|\right)_{x} u_{x} u+\left.\int_{0}^{T}\left|\varphi_{x}\right| u u_{x}\right|_{0} ^{l}\right) \\
& \leq \frac{\lambda}{2 s} \iint\left|\varphi_{x}\right|^{-1}\left|u_{x x}\right|^{2}+\frac{\lambda s^{3}}{2} \iint\left|\varphi_{x}\right|^{3}|u|^{2}+\frac{\lambda s}{2} \iint\left(\left|\varphi_{x}\right|\right)_{x x}|u|^{2}+\left.\lambda s \int_{0}^{T}\left(\left|\varphi_{x}\right| u u_{x}-\left(\left|\varphi_{x}\right|\right)_{x} \frac{|u|^{2}}{2}\right)\right|_{0} ^{l} \\
& \leq \frac{\lambda}{2} A\left(\frac{\left\|M_{1} u\right\|^{2}}{\lambda s}+s^{3} \iint\left|\varphi_{x}\right|^{3} u^{2}\right) \\
&+\frac{\lambda s^{3}}{2} \iint\left|\varphi_{x}\right|^{3}|u|^{2}+\frac{\lambda s}{2} \iint\left(\left|\varphi_{x}\right|\right)_{x x}|u|^{2}+\left.\lambda s \int_{0}^{T}\left(\left|\varphi_{x}\right| u u_{x}-\left(\left|\varphi_{x}\right|\right)_{x} \frac{|u|^{2}}{2}\right)\right|_{0} ^{l} \\
& \leq \frac{A}{2}\left(s^{-1}\left\|M_{1} u\right\|^{2}+s^{3} \lambda \iint\left|\varphi_{x}\right|^{3} u^{2}\right)+\frac{\lambda s^{3}}{2} \iint\left|\varphi_{x}\right|^{3}|u|^{2}+\frac{\lambda s}{2} \iint\left(\left|\varphi_{x}\right|\right)_{x x}|u|^{2} \\
&+\lambda s \int_{0}^{T}\left(\left|\varphi_{x}\right|\left|u \| u_{x}\right|+\left|\left(\left|\varphi_{x}\right|\right)_{x}\right| \frac{|u|^{2}}{2}\right)(0)+\lambda s \int_{0}^{T}\left(\left|\varphi_{x}\right||u|\left|u_{x}\right|+\left|\left(\left|\varphi_{x}\right|\right)_{x}\right| \frac{|u|^{2}}{2}\right)(l)
\end{aligned}
$$

The claim follows by summing (3.17) and (3.18) over all the edges. 
Step 4. Conclusion.

By (3.7), (3.15) and (B1), we get for $\lambda \geq 1$

$$
\begin{aligned}
\|\mathbf{w}\|^{2}= & \left\|M_{1} \mathbf{u}+M_{2} \mathbf{u}\right\|^{2} \\
= & \left\|M_{1} \mathbf{u}\right\|^{2}+\left\|M_{2} \mathbf{u}\right\|^{2}+2\left(M_{1} \mathbf{u}, M_{2} \mathbf{u}\right) \\
= & \left\|M_{1} \mathbf{u}\right\|^{2}+\left\|M_{2} \mathbf{u}\right\|^{2}+2\left\{\sum_{\bar{\alpha} \in I_{\mathcal{J}}} X^{\bar{\alpha}}+Y\right. \\
& \left.-2 s \int_{0}^{T} \int_{\Gamma} \boldsymbol{\varphi}_{x x}\left|\mathbf{u}_{x}\right|^{2}+\int_{0}^{T} \int_{\Gamma}|\mathbf{u}|^{2}\left[\frac{s}{2}\left(\boldsymbol{\varphi}_{4 x}-\boldsymbol{\varphi}_{t t}\right)-s^{2}\left(\left|\boldsymbol{\varphi}_{x}^{2}\right|\right)_{t}-s^{3} \boldsymbol{\varphi}_{x}\left(\left|\boldsymbol{\varphi}_{x}\right|^{2}\right)_{x}\right]\right\} \\
\geq & \left\|M_{1} \mathbf{u}\right\|^{2}+\left\|M_{2} \mathbf{u}\right\|^{2}+2\left\{\sum_{\bar{\alpha} \in I_{\mathcal{J}}} X^{\bar{\alpha}}+Y\right. \\
& \left.+2 s \int_{0}^{T} \int_{\Gamma}\left(\lambda^{2} \boldsymbol{\psi}_{x}^{2}+\lambda \boldsymbol{\psi}_{x x}\right) \theta\left|\mathbf{u}_{x}\right|^{2}+A \lambda s^{3} \int_{0}^{T} \int_{\Gamma}|\mathbf{u}|^{2}\left|\boldsymbol{\varphi}_{x}\right|^{3}\right\} \\
\geq & \left\|M_{1} \mathbf{u}\right\|^{2}+\left\|M_{2} \mathbf{u}\right\|^{2}+\sum_{\bar{\alpha} \in I_{\mathcal{J}}} X^{\bar{\alpha}}+Y+A \lambda s^{3} \int_{0}^{T} \int_{\Gamma}|\mathbf{u}|^{2}\left|\boldsymbol{\varphi}_{x}\right|^{2} .
\end{aligned}
$$

Multiplying (3.16) by $A / 2 C$ and adding it to (3.19) we get

$$
\begin{aligned}
\left\|M_{2} \mathbf{u}\right\|^{2}+ & \left\|M_{1} \mathbf{u}\right\|^{2}\left(1-\frac{A}{2 s}\right)+\frac{A \lambda s^{3}}{2} \iint|\mathbf{u}|^{2}\left|\boldsymbol{\varphi}_{x}\right|^{3}+\frac{A \lambda s}{2 C} \iint\left|\boldsymbol{\varphi}_{x}\right|\left|\mathbf{u}_{x}\right|^{2}+\frac{A \lambda}{2 s C} \iint\left|\boldsymbol{\varphi}_{x}\right|^{-1}\left|\Delta_{\Gamma} \mathbf{u}\right|^{2} \\
& +\sum_{\bar{\alpha} \in I_{\mathcal{J}}} X^{\bar{\alpha}}+Y \leq\|\mathbf{w}\|^{2}+\frac{A}{2} B
\end{aligned}
$$

where

$$
\begin{aligned}
B & =B_{1}+B_{2} \\
& \left.=\lambda s \int_{0}^{T} \sum_{\bar{\alpha} \in I}\left|\varphi_{x}^{\bar{\alpha}} u^{\bar{\alpha}} u_{x}^{\bar{\alpha}}\right|(0)+\left|\varphi_{x}^{\bar{\alpha}} u^{\bar{\alpha}} u_{x}^{\bar{\alpha}}\right|\left(l_{\bar{\alpha}}\right)+\lambda s \int_{0}^{T} \sum_{\bar{\alpha} \in I}\left|\left(\left|\varphi_{x}^{\bar{\alpha}}\right|\right)_{x}\right|\left|u^{\bar{\alpha}}\right|^{2}(0)+\left|\left(\left|\varphi_{x}^{\bar{\alpha}}\right|\right)_{x}\right|\left|u^{\bar{\alpha}}\right|^{2}\left(l_{\bar{\alpha}}\right)\right) .
\end{aligned}
$$

We now prove that for $s$ large enough, the term $B$ is small compared to $\sum_{\bar{\alpha} \in I_{\mathcal{J}}} X^{\bar{\alpha}}$, so that $B$ can be absorbed by the left hand side of (3.20). Using (3.14) and the fact that $\mathbf{u}$ vanishes at the vertices of $\mathcal{E} \cup \mathcal{R}=\partial \Gamma$, we see that

$$
B_{2} \leq C \lambda^{3} s \int_{0}^{T} \sum_{\bar{\alpha} \in I} \theta\left|u\left(\mathcal{O}_{\bar{\alpha}}, t\right)\right|^{2} d t=C \lambda^{3} s \int_{0}^{T} \sum_{\bar{\alpha} \in I_{\mathcal{J}}} \theta\left|u\left(\mathcal{O}_{\bar{\alpha}}, t\right)\right|^{2} d t \leq \frac{C}{s^{2}} \sum_{\bar{\alpha} \in I_{\mathcal{J}}} X^{\bar{\alpha}} .
$$


INVERSE PROBLEM FOR THE HEAT EQUATION AND THE SCHRÖDINGER EQUATION ON A TREE 17

Using again the fact that $\mathbf{u}$ vanishes at the vertices of $\partial \Gamma$, we obtain with (3.14) that

$$
\begin{aligned}
B_{1} & \leq C \lambda s \int_{0}^{T} \sum_{\bar{\alpha} \in I_{\mathcal{J}}}\left|\varphi_{x}^{\bar{\alpha}} u^{\bar{\alpha}}\right|\left(\mathcal{O}_{\bar{\alpha}}, t\right)\left(\left|u_{x}^{\bar{\alpha}}\left(l_{\bar{\alpha}}, t\right)\right|+\sum_{\beta \in\left[\left[1, m_{\bar{\alpha}}\right]\right]}\left|u_{x}^{\overline{\alpha \beta}}(0, t)\right|\right) \\
& \leq C \int_{0}^{T} \sum_{\bar{\alpha} \in I_{\mathcal{J}}}\left((s \lambda)^{2}\left|\varphi_{x}^{\bar{\alpha}}\right|\left|u^{\bar{\alpha}}\right|^{2}\left(\mathcal{O}_{\bar{\alpha}}, t\right)+\left|\varphi_{x}^{\bar{\alpha}}\left(\mathcal{O}_{\bar{\alpha}}, t\right)\right|\left(\left|u_{x}^{\bar{\alpha}}\left(l_{\bar{\alpha}}, t\right)\right|^{2}+\sum_{\beta \in\left[\left[1, m_{\bar{\alpha}}\right]\right]}\left|u_{x}^{\overline{\alpha \beta}}(0, t)\right|^{2}\right) d t\right. \\
& \leq \frac{C}{s} \sum_{\bar{\alpha} \in I_{\mathcal{J}}} X^{\bar{\alpha}}
\end{aligned}
$$

Gathering together (3.20), (3.21) and (3.22), we obtain

$$
\begin{aligned}
\left\|M_{2} \mathbf{u}\right\|^{2}+ & \left\|M_{1} \mathbf{u}\right\|^{2}\left(1-\frac{A}{2 s}\right)+\frac{A \lambda s^{3}}{2} \int_{0}^{T} \int_{\Gamma}|\mathbf{u}|^{2}\left|\boldsymbol{\varphi}_{x}\right|^{3}+\frac{A \lambda s}{2 C} \iint\left|\boldsymbol{\varphi}_{x} \| \mathbf{u}_{x}\right|^{2}+\frac{A \lambda}{2 s C} \int_{0}^{T} \int_{\Gamma}\left|\boldsymbol{\varphi}_{x}\right|^{-1}\left|\Delta_{\Gamma} \mathbf{u}\right|^{2} \\
& +\left(1-\frac{C}{s}\right) \sum_{\bar{\alpha} \in I_{\mathcal{J}}} X^{\bar{\alpha}}+Y \leq\|\mathbf{w}\|^{2} .
\end{aligned}
$$

Writing explicitly the term $Y$ and tacking into account the sign of the functions $\psi_{x}^{\bar{\alpha}}$ occuring in $Y$, we get for $s$ and $\lambda$ large enough

$$
\begin{aligned}
\left\|M_{1} \mathbf{u}\right\|^{2}+ & \left\|M_{2} \mathbf{u}\right\|^{2}+\lambda s^{3} \int_{0}^{T} \int_{\Gamma}|\mathbf{u}|^{2}\left|\boldsymbol{\varphi}_{x}\right|^{3}+\lambda s \int_{0}^{T} \int_{\Gamma}\left|\boldsymbol{\varphi}_{x} \| \mathbf{u}_{x}\right|^{2}+\lambda s^{-1} \int_{0}^{T} \int_{\Gamma}\left|\boldsymbol{\varphi}_{x}\right|^{-1}\left|\Delta_{\Gamma} \mathbf{u}\right|^{2} \\
& +\sum_{\bar{\alpha} \in I_{\mathcal{J}}} X^{\bar{\alpha}}+\int_{0}^{T} \lambda s \theta^{\overline{1}}\left|u_{x}\right|^{2}(\mathcal{R}, t) d t \leq C\left(\|\mathbf{w}\|^{2}+\int_{0}^{T} \sum_{\bar{\alpha} \in I_{\varepsilon}} \lambda s \theta^{\bar{\alpha}}\left|u_{x}^{\bar{\alpha}}\right|^{2}\left(l_{\bar{\alpha}}, t\right) d t\right) .
\end{aligned}
$$

Finally, using the definition of $M_{2}$ we get

$$
\begin{aligned}
\lambda s^{-1} \int_{0}^{T} \int_{\Gamma}\left|\boldsymbol{\varphi}_{x}\right|^{-1}\left|\mathbf{u}_{t}\right|^{2} d x d t & \leq C \lambda s^{-1} \int_{0}^{T} \int_{\Gamma}\left|\boldsymbol{\varphi}_{x}\right|^{-1}\left(\left|M_{2} \mathbf{u}\right|^{2}+s^{2}\left|\boldsymbol{\varphi}_{x}\right|^{2}\left|\mathbf{u}_{x}\right|^{2}+s^{2}\left|\boldsymbol{\varphi}_{x x}\right|^{2}|\mathbf{u}|^{2}\right) \\
& \leq C \int_{0}^{T} \int_{\Gamma} s^{-1}\left|M_{2} \mathbf{u}\right|^{2}+\lambda s\left|\boldsymbol{\varphi}_{x}\right|\left|\mathbf{u}_{x}\right|^{2}+\lambda s\left|\boldsymbol{\varphi}_{x}\right|^{-1}\left|\boldsymbol{\varphi}_{x x}\right|^{2}|\mathbf{u}|^{2}(\beta .24)
\end{aligned}
$$

From (3.23) and (3.24), we infer that for $s \geq s_{3}$ and $\lambda \geq \lambda_{3}$ (with $s_{3}, \lambda_{3}$ large enough) we have that

$$
\begin{aligned}
\left\|M_{1} \mathbf{u}\right\|^{2}+ & \left\|M_{2} \mathbf{u}\right\|^{2}+\lambda s^{3} \int_{0}^{T} \int_{\Gamma}|\mathbf{u}|^{2}\left|\boldsymbol{\varphi}_{x}\right|^{3}+\lambda s \int_{0}^{T} \int_{\Gamma}\left|\boldsymbol{\varphi}_{x} \| \mathbf{u}_{x}\right|^{2}+\lambda s^{-1} \int_{0}^{T} \int_{\Gamma}\left|\boldsymbol{\varphi}_{x}\right|^{-1}\left(\left|\Delta_{\Gamma} \mathbf{u}\right|^{2}+\left|\mathbf{u}_{t}\right|^{2}\right) \\
& +\sum_{\bar{\alpha} \in I_{\mathcal{J}}} X^{\bar{\alpha}}+\int_{0}^{T} \lambda s \theta^{\overline{1}}\left|u_{x}\right|^{2}(\mathcal{R}, t) d t \leq C\left(\|\mathbf{w}\|^{2}+\int_{0}^{T} \sum_{\bar{\alpha} \in I_{\mathcal{E}}} \lambda s \theta^{\bar{\alpha}}\left|u_{x}^{\bar{\alpha}}\right|^{2}\left(l_{\bar{\alpha}}, t\right) d t\right) .
\end{aligned}
$$

Replacing $\mathbf{u}$ by $e^{-s \varphi} \mathbf{q}$ in the last inequality, we readily obtain (3.3). 
3.3. Inverse problem. Before proving the stability result in Theorem 1.1 we need to analyze the following system:

$$
\left\{\begin{array}{lll}
u_{t}^{\bar{\alpha}}(x, t)=u_{x x}^{\bar{\alpha}}(x, t)+b^{\bar{\alpha}}(x) u^{\bar{\alpha}}(x, t)+R^{\bar{\alpha}}(x, t) f^{\bar{\alpha}}(x), & & (x, t) \in\left(0, l_{\bar{\alpha}}\right) \times(0, T), \alpha \in I, \\
u^{\bar{\alpha}}\left(l_{\bar{\alpha}}, t\right)=0, & & t \in(0, T), \alpha \in I_{\mathcal{E}}, \\
u^{\overline{1}}(0, t)=0, & & t \in(0, T), \\
u^{\bar{\alpha}}\left(l_{\bar{\alpha}}, t\right)=u^{\overline{\alpha \beta}}(0, t), & t \in(0, T), \alpha \in I_{\mathcal{J}}, \beta \in\left[\left[1, m_{\bar{\alpha}}\right]\right], \\
u_{x}^{\bar{\alpha}}\left(l_{\bar{\alpha}}, t\right)=\sum_{\beta=1}^{m_{\bar{\alpha}}} u_{x}^{\overline{\alpha \beta}}(0, t), & t \in(0, T), \bar{\alpha} \in I_{\mathcal{J}},
\end{array}\right.
$$

where $\mathbf{b}=\left\{b^{\bar{\alpha}}\right\}_{\bar{\alpha} \in I} \in L^{\infty}(\Gamma)$.

Proposition 3.4. Assume that $\mathbf{u}=\left\{u^{\bar{\alpha}}\right\}_{\bar{\alpha} \in I}$ is a solution of (3.25) which satisfies $\mathbf{u}_{t} \in H^{2,1}(\Gamma \times$ $(0, T))$. If $\mathbf{R}=\left\{R^{\bar{\alpha}}(x, t)\right\}_{\bar{\alpha} \in I}$ is such that $\mathbf{R}_{t} \in L^{\infty}(\Gamma \times(0, T))$ and that

$$
\left|\mathbf{R}\left(x, t_{0}\right)\right| \geq r>0, \quad \text { for a.e. } x \in \Gamma \text { and some } t_{0} \in(0, T),
$$

then there exists a positive constant $C=C\left(\left\|\mathbf{R}_{t}\right\|_{L^{\infty}(\Gamma \times(0, T))},\|\mathbf{b}\|_{L^{\infty}(\Gamma)}, r\right)$ such that

$$
\|\mathbf{f}\|_{L^{2}(\Gamma)} \leq C\left(\left\|\mathbf{u}\left(\cdot, t_{0}\right)\right\|_{H^{2}(\Gamma)}+\sum_{v \in \mathcal{E}}\left\|\partial_{x t} \mathbf{u}(v, \cdot)\right\|_{L^{2}(0, T)}\right)
$$

for any $\mathbf{f} \in L^{2}(\Gamma)$.

Proof. We proceed as in [34]. Set $\mathbf{z}=\partial_{t} \mathbf{u}$. Then $\mathbf{z}=\left\{z^{\bar{\alpha}}\right\}_{\bar{\alpha} \in I}$ satisfies

$$
\left\{\begin{array}{lll}
z_{t}^{\bar{\alpha}}(x, t)=z_{x x}^{\bar{\alpha}}(x, t)+b^{\bar{\alpha}}(x) z^{\bar{\alpha}}(x, t)+R_{t}^{\bar{\alpha}}(x, t) f^{\bar{\alpha}}(x), & & (x, t) \in\left(0, l_{\bar{\alpha}}\right) \times(0, T), \alpha \in I, \\
z^{\bar{\alpha}}\left(l_{\bar{\alpha}}, t\right)=0, & & t \in(0, T), \alpha \in I_{\mathcal{E}}, \\
z^{\overline{1}}(0, t)=0, & & t \in(0, T), \\
z^{\bar{\alpha}}\left(l_{\bar{\alpha}}, t\right)=z^{\overline{\alpha \beta}}(0, t), & & t \in(0, T), \alpha \in I_{\mathcal{J}}, \beta \in\left[\left[1, m_{\bar{\alpha}}\right]\right], \\
z_{x}^{\bar{\alpha}}\left(l_{\bar{\alpha}}, t\right)=\sum_{\beta=1}^{m_{\bar{\alpha}}} z_{x}^{\overline{\alpha \beta}}(0, t), &
\end{array}\right.
$$

On the other hand,

$$
\mathbf{R}(x, t) \mathbf{f}(x)=\partial_{t} \mathbf{u}(x, t)-\left(\Delta_{\Gamma} \mathbf{u}\right)(x, t)-\mathbf{b u}(x, t) .
$$

Using a change of variables it is sufficient to prove (3.27) when $t_{0}=T / 2$. 
We now apply the Carleman estimate (3.3) with $\mathbf{q}=\mathbf{z}=\partial_{t} \mathbf{u}$ (and some fixed $\lambda>0$ )

$$
\begin{gathered}
\int_{0}^{T} \int_{\Gamma}\left[(s \boldsymbol{\theta})^{-1}\left|\partial_{t t} \mathbf{u}\right|^{2}+s \boldsymbol{\theta}\left|\partial_{x t} \mathbf{u}\right|^{2}+(s \boldsymbol{\theta})^{3}\left|\partial_{t} \mathbf{u}\right|^{2}\right] e^{-2 s \boldsymbol{\varphi}}+\int_{0}^{T}(s \boldsymbol{\theta})\left(\left|\partial_{x t} \mathbf{u}\right|^{2} e^{-2 s \boldsymbol{\varphi}}\right)(\mathcal{R}, t) d t \\
\leq C\left(\int_{0}^{T} \int_{\Gamma}\left|\partial_{t t} \mathbf{u}-\Delta_{\Gamma} \partial_{t} \mathbf{u}\right|^{2} e^{-2 s \boldsymbol{\varphi}}+\sum_{v \in \mathcal{E}} \int_{0}^{T} s \boldsymbol{\theta}\left(\left|\partial_{x t} \mathbf{u}\right|^{2} e^{-2 s \boldsymbol{\varphi}}\right)(v, t) d t\right) \\
\leq C\left(\int_{0}^{T} \int_{\Gamma}\left(\left|\left(\partial_{t} \mathbf{R}\right) \mathbf{f}\right|^{2}+\left|\partial_{t} \mathbf{u}\right|^{2}\right) e^{-2 s \boldsymbol{\varphi}}+\sum_{v \in \mathcal{E}} \int_{0}^{T} s \boldsymbol{\theta}\left(\left|\partial_{x t} \mathbf{u}\right|^{2} e^{-2 s \boldsymbol{\varphi}}\right)(v, t) d t\right),
\end{gathered}
$$

which gives, for $s$ large enough,

$$
\begin{gathered}
\int_{0}^{T} \int_{\Gamma}\left[(s \boldsymbol{\theta})^{-1}\left|\partial_{t t} \mathbf{u}\right|^{2}+s \boldsymbol{\theta}\left|\partial_{x t} \mathbf{u}\right|^{2}+(s \boldsymbol{\theta})^{3}\left|\partial_{t} \mathbf{u}\right|^{2}\right] e^{-2 s \boldsymbol{\varphi}}+\int_{0}^{T}(s \boldsymbol{\theta})\left(\left|\partial_{x t} \mathbf{u}\right|^{2} e^{-2 s \boldsymbol{\varphi}}\right)(\mathcal{R}, t) d t \\
\leq C\left(\int_{0}^{T} \int_{\Gamma}\left|\left(\partial_{t} \mathbf{R}\right) \mathbf{f}\right|^{2} e^{-2 s \boldsymbol{\varphi}}+\sum_{v \in \mathcal{E}} \int_{0}^{T} s \boldsymbol{\theta}\left(\left|\partial_{x t} \mathbf{u}\right|^{2} e^{-2 s \boldsymbol{\varphi}}\right)(v, t) d t\right) .
\end{gathered}
$$

Since $\lim _{t \rightarrow 0} e^{-2 s \boldsymbol{\varphi}(x, t)}=0$ for $x \in \Gamma$ and $\left|\boldsymbol{\varphi}_{t}(x, t)\right| \leq C \boldsymbol{\theta}^{2}(x, t)$ for all $x \in \Gamma$ and $t>0$, we get

$$
\begin{aligned}
\int_{\Gamma}\left|\partial_{t} \mathbf{u}\left(x, \frac{T}{2}\right)\right|^{2} e^{-2 s \boldsymbol{\varphi}\left(x, \frac{T}{2}\right)} d x=\int_{0}^{T / 2} \frac{\partial}{\partial t}\left(\int_{\Gamma}\left|\partial_{t} \mathbf{u}(x, t)\right|^{2} e^{-2 s \boldsymbol{\varphi}(x, t)} d x\right) d t \\
\quad=\int_{0}^{T / 2} \int_{\Gamma}\left[2 \partial_{t} \mathbf{u} \partial_{t t} \mathbf{u}-2 s \partial_{t} \boldsymbol{\varphi}\left|\partial_{t} \mathbf{u}\right|^{2}\right] e^{-2 s \boldsymbol{\varphi}(x, t)} d x d t \\
\leq \int_{0}^{T / 2} \int_{\Gamma}\left(2\left|\partial_{t} \mathbf{u}\right|\left|\partial_{t t} \mathbf{u}\right|+C s \boldsymbol{\theta}^{2}\left|\partial_{t} \mathbf{u}\right|^{2}\right) e^{-2 s \boldsymbol{\varphi}(x, t)} d x d t \\
\leq C \int_{0}^{T / 2} \int_{\Gamma}\left(\left(s^{2} \boldsymbol{\theta}\right)^{-1}\left|\partial_{t t} \mathbf{u}\right|^{2}+(s \boldsymbol{\theta})^{2}\left|\partial_{t} \mathbf{u}\right|^{2}\right) e^{-2 s \boldsymbol{\varphi}(x, t)} d x d t \\
\leq \frac{C}{s} \int_{0}^{T} \int_{\Gamma}\left|\left(\partial_{t} \mathbf{R}\right) \mathbf{f}\right|^{2} e^{-2 s \boldsymbol{\varphi}} d x d t+C e^{-C s} \sum_{v \in \mathcal{E}} \int_{0}^{T}\left|\partial_{x t} \mathbf{u}\right|^{2}(v, t) d t
\end{aligned}
$$

Using (3.26), (3.29) and (3.30) we obtain that

$$
\begin{aligned}
\int_{\Gamma}|\mathbf{f}(x)|^{2} & e^{-2 s \boldsymbol{\varphi}(x, T / 2)} d x \leq C \int_{\Gamma}|\mathbf{R}(x, T / 2) \mathbf{f}(x)|^{2} e^{-2 s \boldsymbol{\varphi}(x, T / 2)} d x \\
& \leq C \int_{\Gamma}\left(\left|\partial_{t} \mathbf{u}\left(x, \frac{T}{2}\right)\right|^{2}+\left|\Delta_{\Gamma} \mathbf{u}\left(x, \frac{T}{2}\right)\right|^{2}+\left|\mathbf{u}\left(x, \frac{T}{2}\right)\right|^{2}\right) e^{-2 s \boldsymbol{\varphi}(x, T / 2)} d x \\
& \leq \frac{C}{s} \int_{0}^{T} \int_{\Gamma}\left|\left(\partial_{t} \mathbf{R}\right) \mathbf{f}\right|^{2} e^{-2 s \boldsymbol{\varphi}} d x d t+C e^{-C s} \sum_{v \in \mathcal{E}} \int_{0}^{T}\left|\partial_{x t} \mathbf{u}\right|^{2}(v, t) d t+C\left\|\mathbf{u}\left(\cdot, \frac{T}{2}\right)\right\|_{H^{2}(\Gamma)}^{2} \cdot
\end{aligned}
$$


Since $\partial_{t} \mathbf{R} \in L^{\infty}(\Gamma \times(0, T))$, it holds

$$
\begin{aligned}
\int_{\Gamma}|\mathbf{f}(x)|^{2} & e^{-2 s \boldsymbol{\varphi}(x, T / 2)} d x \\
& \leq \frac{C}{s} \int_{0}^{T} \int_{\Gamma}|\mathbf{f}|^{2} e^{-2 s \boldsymbol{\varphi}} d x d t+C e^{-C s} \sum_{v \in \mathcal{E}} \int_{0}^{T}\left|\partial_{x t} \mathbf{u}\right|^{2}(v, t) d t+C\left\|\mathbf{u}\left(\cdot, \frac{T}{2}\right)\right\|_{H^{2}(\Gamma)}^{2} .
\end{aligned}
$$

It follows from the definition of $\varphi$ that

$$
\varphi\left(x, \frac{T}{2}\right) \leq \varphi(x, t) \quad \text { for all }(x, t) \in \Gamma \times(0, T),
$$

so that

$$
\int_{0}^{T} \int_{\Gamma}|\mathbf{f}(x)|^{2} e^{-2 s \varphi(x, t)} d x d t \leq T \int_{\Gamma}|\mathbf{f}(x)|^{2} e^{-2 s \varphi(x, T / 2)} d t
$$

Therefore

$$
\left(1-\frac{C T}{s}\right) \int_{\Gamma}|\mathbf{f}(x)|^{2} e^{-2 s \boldsymbol{\varphi}(x, T / 2)} d t \leq C \sum_{v \in \mathcal{E}} \int_{0}^{T}\left|\partial_{x t} \mathbf{u}\right|^{2}(v, t) d t+C\left\|\mathbf{u}\left(\cdot, \frac{T}{2}\right)\right\|_{H^{2}(\Gamma)}^{2} .
$$

The desired inequality follows for $s$ large enough.

We are now able to prove the stability result for system (1.1).

Proof of Theorem 1.1. Let us denote

$$
\mathbf{w}=\mathbf{u}(\mathbf{p})-\mathbf{u}(\mathbf{q})
$$

It satisfies the following system

$$
\begin{cases}\mathbf{w}_{t}=\Delta_{\Gamma} \mathbf{w}-\mathbf{q w}+\mathbf{R f} & \text { in } \Gamma \times(0, T) \\ \mathbf{w}(x, t)=0, & \text { on } \partial \Gamma \times(0, T),\end{cases}
$$

where $\mathbf{f}=\mathbf{q}-\mathbf{p}, \mathbf{R}=\mathbf{u}(\mathbf{p})$. Note that $\mathbf{R} \in C\left([0, T] ; H^{1}(\Gamma)\right) \subset C(\bar{\Gamma} \times[0, T])$, for $u \in$ $L^{2}\left(0, T ; H^{2}(\Gamma)\right)$ and $u_{t} \in L^{2}\left(0, T ; L^{2}(\Gamma)\right)$. Using our hypothesis, we see that $\left|\mathbf{R}\left(\cdot, t_{0}\right)\right| \geq r>0$ on $\Gamma$, hence we can apply Proposition 3.4 to obtain

$$
\|\mathbf{p}-\mathbf{q}\|_{L^{2}(\Gamma)} \leq C\left(\left\|[\mathbf{u}(\mathbf{p})-\mathbf{u}(\mathbf{q})]\left(\cdot, t_{0}\right)\right\|_{\left.H^{2}(\Gamma)\right)}+\sum_{v \in \mathcal{E}}\left\|\partial_{x}[\mathbf{u}(\mathbf{p})-\mathbf{u}(\mathbf{q})](v, \cdot)\right\|_{H^{1}(0, T)}\right),
$$

where $C=C\left(\left\|\partial_{t} \mathbf{u}(\mathbf{p})\right\|_{L^{\infty}(\Gamma \times(0, T))},\|\mathbf{q}\|_{L^{\infty}(\Gamma)}, r\right)$. The proof is now completed.

\section{Schrödinger EQUATION ON A STAR-ShAPEd TREE}

In this section, we consider a network $\Gamma$ which is a star-shaped tree constituted by $N$ edges $e_{j}$ (with $N \geq 3$ ) connected at the internal node $\mathcal{O}$. Here, the parameterization of the edge $e_{j}$ is chosen so that the origin $\mathcal{O}$ of $e_{j}$ corresponds to $x=0$, while the endpoint $\mathcal{O}_{j}$ of $e_{j}$ corresponds to $x=l_{j}$, for all $j \in[[1, N]]$. 


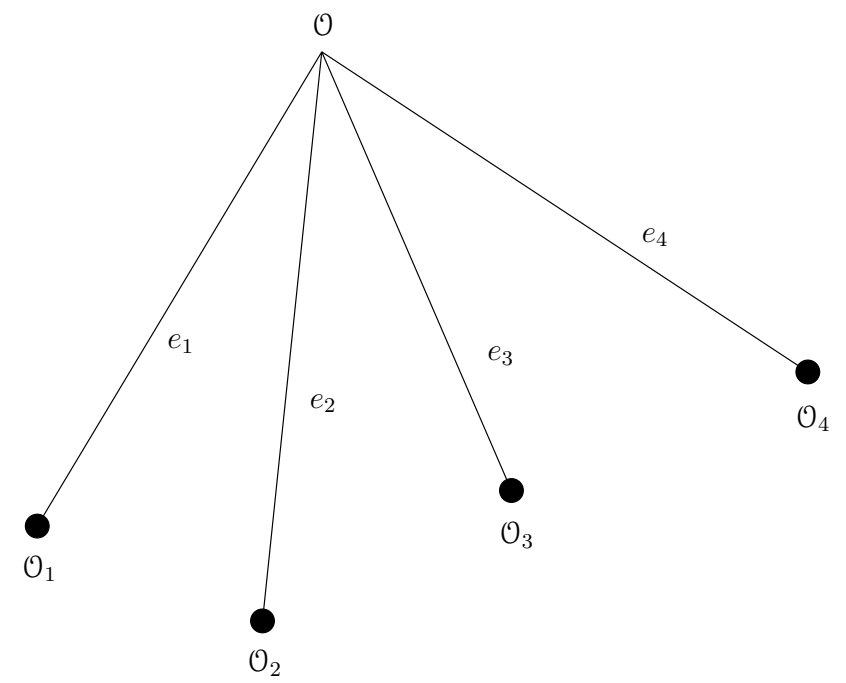

FiguRE 2. A star-shaped tree with 4 edges

We consider the following Cauchy problem

$$
\begin{aligned}
i y_{j, t}+y_{j, x x}+p_{j}(x) y_{j}=f_{j}(x, t), & x \in\left(0, l_{j}\right), j \in[[1, N]], t \in(0, T), \\
y_{j}(0, t)=y_{l}(0, t), & t \in(0, T), j, l \in[[1, N]], \\
\sum_{1 \leq j \leq N} y_{j, x}(0, t)=0, & t \in(0, T), \\
y\left(l_{j}, t\right)=0, & j \in[[1, N]], t \in(0, T), \\
y(x, 0)=y_{0}(x), & x \in \Gamma,
\end{aligned}
$$

where $\mathbf{p}=\left\{p_{j}\right\}_{j=1, N} \in L^{\infty}(\Gamma)$ is some given potential function. Our main aim is to prove the stability for the inverse problem consisting in retrieving the potential $\mathbf{p}$ from the measurement of $y_{x}\left(l_{j}, t\right)$ for $j \in[[1, N]]$. This is done thanks to some Carleman estimate in following the classical Bukhgeim-Klibanov method.

The first step will be the proof of a Carleman inequality on $\Gamma$. The key point is that choosing only one weight function $\boldsymbol{\psi}=\left\{\psi_{j}\right\}_{j=1, N}: \Gamma \rightarrow \mathbb{R}$ as in the case of the heat equation is not convenient since we fail to control some boundary terms. Instead, we consider a family of weights $\left\{\boldsymbol{\psi}^{k}\right\}_{k=1, N}$ allowing us to get rid of some bad boundary terms. 
4.1. Carleman estimate. Assume given a family $\left(\psi_{j}^{k}\right)_{1 \leq j, k \leq N}$ of functions fulfilling the following properties

$$
\begin{aligned}
\psi_{j}^{k}:\left[0, l_{j}\right] \rightarrow \mathbb{R} \text { is of class } C^{2}, & \forall j, k \in[[1, N]], \\
\psi_{j_{1}}^{k_{1}}(0)=\psi_{j_{2}}^{k_{2}}(0), & \forall j_{1}, k_{1}, j_{2}, k_{2} \in[[1, N]], \\
\left|\left(\psi_{j}^{k}\right)^{\prime}(x)\right|^{2}+\left(\psi_{j}^{k}\right)^{\prime \prime}(x) \geq 0, & \forall x \in\left[0, l_{j}\right], \forall j, k \in[[1, N]], \\
\left(\psi_{j}^{k}\right)^{\prime}(x) \neq 0, & \forall x \in\left[0, l_{j}\right], \forall j, k \in[[1, N]], \\
\frac{C}{2} \geq \psi_{j}^{k}(x)>\frac{C}{3}, & \forall x \in\left[0, l_{j}\right], \forall j, k \in[[1, N]],
\end{aligned}
$$

where $C>0$ is some positive constant. We also assume that the following flux conditions at $x=0$ are satisfied:

$$
\begin{aligned}
\sum_{1 \leq j \leq N}\left(\psi_{j}^{k}\right)^{\prime}(0)=0, & \forall k \in[[1, N]], \\
\sum_{1 \leq k \leq N}\left(\psi_{j}^{k}\right)^{\prime}(0)=0, & \forall j \in[[1, N]], \\
\sum_{1 \leq k \leq N}\left|\left(\psi_{j}^{k}\right)^{\prime}(0)\right|^{2}=C_{1}, & \forall j \in[[1, N]], \\
\sum_{1 \leq k \leq N}\left(\psi_{j}^{k}\right)^{\prime \prime}(0)=C_{2}, & \forall j \in[[1, N]], \\
\sum_{1 \leq k \leq N}\left[\left(\psi_{j}^{k}\right)^{\prime}(0)\right]^{3}>0, & \forall j \in[[1, N]],
\end{aligned}
$$

for some constants $C_{1}>0$ and $C_{2} \in \mathbb{R}$. Such a family of weights functions $\left(\psi_{j}^{k}\right)_{1 \leq j, k \leq N}$ exists. It is sufficient to pick (affine) functions of the form $\psi_{j}^{k}(x)=a_{j}^{k} x+\frac{5}{12} C$ with $C>>1$ and

$$
a_{j}^{k}:= \begin{cases}N-1 & \text { if } j=k \\ -1 & \text { if } j \neq k\end{cases}
$$

Let us introduce the families of weights

$$
\theta_{j}^{k}(x, t)=\frac{e^{\lambda \psi_{j}^{k}(x)}}{t(T-t)}, \quad \varphi_{j}^{k}(x, t)=\frac{e^{\lambda C}-e^{\lambda \psi_{j}^{k}(x)}}{t(T-t)}
$$

and the class of functions

$z=\left\{\mathbf{q}=\left(q_{j}\right)_{j=1, N} \in C(\Gamma \times[0, T]) ; q_{j} \in C^{2,1}\left(\left[0, l_{j}\right] \times[0, T]\right) \forall j \in[[1, N]]\right.$, and (4.32)-(4.34) hold $\}$.

Proposition 4.1. Assume that the family of weights $\left(\psi_{k}^{j}\right)$ fulfills (4.36)-(4.45). Then there exist some constants $\lambda_{0} \geq 1, s_{0} \geq 1$ and $C_{0}>0$ such that for all $\lambda \geq \lambda_{0}$, all $s \geq s_{0}$, and all $\mathbf{q} \in Z$, it 
holds

$$
\begin{aligned}
& \sum_{1 \leq j, k \leq N} \int_{0}^{T} \int_{0}^{l_{j}}\left[\lambda^{2} s \theta_{j}^{k}\left|q_{j, x}\right|^{2}+\lambda^{4}\left(s \theta_{j}^{k}\right)^{3}\left|q_{j}\right|^{2}+\left|\left(\widetilde{M}_{1}^{k} \mathbf{q}\right)_{j}\right|^{2}+\left|\left(\widetilde{M}_{2}^{k} \mathbf{q}\right)_{j}\right|^{2}\right] e^{-2 s \varphi_{j}^{k}} d x d t \\
\leq & C_{0} \sum_{1 \leq j, k \leq N}\left(\int_{0}^{T} \int_{0}^{l_{j}}\left|q_{j, t}+i q_{j, x x}\right|^{2} e^{-2 s \varphi_{j}^{k}} d x d t+\int_{0}^{T} \lambda s \theta_{j}^{k}\left(l_{j}\right)\left|q_{j, x}\left(l_{j}\right)\right|^{2} e^{-2 s \varphi_{j}^{k}} d t\right),
\end{aligned}
$$

where $i=\sqrt{-1}$ and $\widetilde{M}_{1}^{k}$ and $\widetilde{M}_{2}^{k}$ denote the operators

$$
\begin{aligned}
&\left(\widetilde{M}_{1}^{k} \mathbf{q}\right)_{j}:=\left[s\left(\varphi_{j, t}^{k}+i \varphi_{j, x x}^{k}\right)-2 i s^{2}\left|\varphi_{j, x}^{k}\right|^{2}\right] q_{j}+2 i s \varphi_{j, x}^{k} q_{j, x}, \\
&\left(\widetilde{M}_{2}^{k} \mathbf{q}\right)_{j}:=\left[-s\left(\varphi_{j, t}^{k}+i \varphi_{j, x x}^{k}\right)+2 i s^{2}\left|\varphi_{j, x}^{k}\right|^{2}\right] q_{j}+q_{j, t}-2 i s \varphi_{j, x}^{k} q_{j, x}+i q_{j, x x} .
\end{aligned}
$$

Proof. In what follows, the letter $c$ will denote a constant (independent of $s, \lambda, \mathbf{q}, j, k$ ) which may vary from line to line. Let $\mathbf{q} \in \mathcal{Z}$ be given, and for $j, k \in[[1, N]]$, let

$$
u_{j}^{k}=e^{-s \varphi_{j}^{k}} q_{j}, \quad w_{j}^{k}=e^{-s \varphi_{j}^{k}} L\left(e^{s \varphi_{j}^{k}} u_{j}^{k}\right)
$$

where $L$ denotes the operator

$$
L=\partial_{t}+i \partial_{x}^{2} .
$$

Straightforward computations show that $\mathbf{w}^{k}=M^{k} \mathbf{u}^{k}$ with

$$
w_{j}^{k}=\left(M^{k} \mathbf{u}^{k}\right)_{j}:=u_{j, t}^{k}+s \varphi_{j, t}^{k} u_{j}^{k}+i\left(u_{j, x x}^{k}+2 s \varphi_{j, x}^{k} u_{j, x}^{k}+s \varphi_{j, x x}^{k} u_{j}^{k}+s^{2}\left|\varphi_{j, x}^{k}\right|^{2} u_{j}^{k}\right),
$$

the operator $M^{k}$ acting simply on the components of $\mathbf{u}^{k}$ along the different edges. Let $M_{1}^{k}$ and $M_{2}^{k}$ denote respectively the (formal) adjoint and skew-adjoint parts of the operator $M^{k}$. We readily obtain that

$$
\begin{aligned}
\left(M_{1}^{k} \mathbf{u}^{k}\right)_{j} & =i\left(2 s \varphi_{j, x}^{k} u_{j, x}^{k}+s \varphi_{j, x x}^{k} u_{j}^{k}\right)+s \varphi_{j, t}^{k} u_{j}^{k} \\
\left(M_{2}^{k} \mathbf{u}^{k}\right)_{j} & =u_{j, t}^{k}+i\left(u_{j, x x}^{k}+s^{2}\left|\varphi_{j, x}^{k}\right|^{2} u_{j}^{k}\right) .
\end{aligned}
$$

Letting $\left(\widetilde{M_{1}^{k}} \mathbf{q}\right)_{j}:=e^{s \varphi_{j}^{k}}\left(M_{1}^{k} \mathbf{u}^{k}\right)_{j}$ and $\left(\widetilde{M}_{2}^{k} \mathbf{q}\right)_{j}:=e^{s \varphi_{j}^{k}}\left(M_{2}^{k} \mathbf{u}^{k}\right)_{j}$, we easily check that (4.47) and (4.48) hold. On the other hand,

$$
\left\|\mathbf{w}^{k}\right\|^{2}=\left\|M_{1}^{k} \mathbf{u}^{k}+M_{2}^{k} \mathbf{u}^{k}\right\|^{2}=\left\|M_{1}^{k} \mathbf{u}^{k}\right\|^{2}+\left\|M_{2}^{k} \mathbf{u}^{k}\right\|^{2}+2 \operatorname{Re}\left(M_{1}^{k} \mathbf{u}^{k}, M_{2}^{k} \mathbf{u}^{k}\right)
$$

where $(\mathbf{u}, \mathbf{v}):=\sum_{1 \leq j \leq N} \int_{0}^{T} \int_{0}^{l_{j}} u_{j}(x, t) \overline{v_{j}(x, t)} d x d t$ and $\|\mathbf{w}\|^{2}=(\mathbf{w}, \mathbf{w})$. The proof of the Carleman estimate is inspired by those of [30, Proposition 2.1]. In the first step, we compute precisely $\operatorname{Re}\left(M_{1}^{k} \mathbf{u}^{k}, M_{2}^{k} \mathbf{u}^{k}\right)$. In the second step, we check that the boundary terms related to the internal node $\mathcal{O}$ give positive contributions. The third step is completely similar to the second step in the proof of [30, Proposition 2.1].

Step 1. Exact computation of $\operatorname{Re}\left(M_{1}^{k} \mathbf{u}^{k}, M_{2}^{k} \mathbf{u}^{k}\right)$.

We intend to compute

$$
\operatorname{Re}\left(M_{1}^{k} \mathbf{u}^{k}, M_{2}^{k} \mathbf{u}^{k}\right)=\operatorname{Re} \sum_{1 \leq j \leq N} \int_{0}^{T} \int_{0}^{l_{j}}\left(M_{1}^{k} u^{k}\right)_{j} \overline{\left(M_{2}^{k} u^{k}\right)_{j}} d x d t .
$$


Let us fix any pair $(j, k)$ of indices in $[[1, N]]$ and let us compute $\int_{0}^{T} \int_{0}^{l_{j}}\left(M_{1}^{k} u^{k}\right)_{j} \overline{\left(M_{2}^{k} u^{k}\right)_{j}} d x d t$. For the sake of simplicity, we shall drop the indices $j$ and $k$ during the computations, and we shall write $\iint u$ for $\int_{0}^{T} \int_{0}^{l_{j}} u(x, t) d x d t$, and $\int h$ for $\int_{0}^{T} h(t) d t$. Then

$\begin{aligned} 2 \operatorname{Re} \int_{0}^{T} \int_{0}^{l_{j}}\left(M_{1}^{k} u^{k}\right)_{j} \overline{\left(M_{2}^{k} u^{k}\right)_{j}} d x d t & =2 \operatorname{Re} \iint\left[i\left(2 s \varphi_{x} u_{x}+s \varphi_{x x} u\right)+s \varphi_{t} u\right]\left[\overline{u_{t}}-i\left(\overline{u_{x x}}+s^{2}\left|\varphi_{x}\right|^{2} \bar{u}\right)\right] \\ & =I_{1}+I_{2}+I_{3}\end{aligned}$

where

$$
\begin{aligned}
I_{1} & =2 \operatorname{Re} \iint i\left(2 s \varphi_{x} u_{x}+s \varphi_{x x} u\right)\left(\overline{u_{t}}-i\left(\overline{u_{x x}}+s^{2}\left|\varphi_{x}\right|^{2} \bar{u}\right)\right) \\
I_{2} & =2 \operatorname{Re} \iint s \varphi_{t} u\left(\overline{u_{t}}-i \overline{u_{x x}}\right) \\
I_{3} & =2 \operatorname{Re} \iint s \varphi_{t} u\left(-i s^{2}\left|\varphi_{x}\right|^{2} \bar{u}\right) .
\end{aligned}
$$

Obviously, $I_{3}=0$. Let us begin with the computation of $I_{1}$.

$$
\begin{aligned}
I_{1}=2 \operatorname{Re} & \iint\left(2 s \varphi_{x} u_{x}+s \varphi_{x x} u\right)\left(\overline{u_{x x}}+s^{2}\left|\varphi_{x}\right|^{2} \bar{u}\right) \\
& +2 \operatorname{Re} \iint i\left(2 s \varphi_{x} u_{x}+s \varphi_{x x} u\right) \overline{u_{t}}=I_{1}^{1}+I_{1}^{2} .
\end{aligned}
$$

To calculate $I_{1}^{1}$, we need to evaluate the real part of the integral term $J:=\iint \overline{u_{x x}} \varphi_{x} u_{x}$. Integrating by part yields

$$
J=-\iint \overline{u_{x}}\left(\varphi_{x x} u_{x}+\varphi_{x} u_{x x}\right)+\left.\int \varphi_{x}\left|u_{x}\right|^{2}\right|_{0} ^{l}
$$

where $l$ stands for $l_{j}$. On the other hand

$$
\begin{aligned}
2 \operatorname{Re} \iint \overline{u_{x}} \varphi_{x} u_{x x} & =\iint \varphi_{x}\left(\overline{u_{x}} u_{x x}+u_{x} \overline{u_{x x}}\right)=\iint \varphi_{x} \partial_{x}\left|u_{x}\right|^{2} \\
& =-\iint \varphi_{x x}\left|u_{x}\right|^{2}+\left.\int \varphi_{x}\left|u_{x}\right|^{2}\right|_{0} ^{l} .
\end{aligned}
$$

Therefore

$$
\begin{aligned}
2 \operatorname{Re} J & =-2 \iint \varphi_{x x}\left|u_{x}\right|^{2}+\iint \varphi_{x x}\left|u_{x}\right|^{2}-\left.\int \varphi_{x}\left|u_{x}\right|^{2}\right|_{0} ^{l}+\left.2 \int \varphi_{x}\left|u_{x}\right|^{2}\right|_{0} ^{l} \\
& =-\iint \varphi_{x x}\left|u_{x}\right|^{2}+\left.\int \varphi_{x}\left|u_{x}\right|^{2}\right|_{0} ^{l}
\end{aligned}
$$


It follows that

$$
\begin{aligned}
I_{1}^{1}= & 2 \operatorname{Re}\left\{2 s J+s \iint \varphi_{x x} u \overline{u_{x x}}+2 s^{3} \iint\left(\varphi_{x}\right)^{3} u_{x} \bar{u}+s^{3} \iint \varphi_{x x}\left|\varphi_{x}\right|^{2}|u|^{2}\right\} \\
= & 4 s \operatorname{Re} J-2 s \operatorname{Re} \iint\left(\varphi_{3 x} u+\varphi_{x x} u_{x}\right) \overline{u_{x}}+\left.2 s \operatorname{Re} \int \varphi_{x x} u \overline{u_{x}}\right|_{0} ^{l}+2 s^{3} \iint\left(\varphi_{x}\right)^{3} \partial_{x}|u|^{2} \\
& \quad+2 s^{3} \iint \varphi_{x x}\left|\varphi_{x}\right|^{2}|u|^{2} \\
= & \left.2 s\left\{-\iint \varphi_{x x}\left|u_{x}\right|^{2}+\left.\int \varphi_{x}\left|u_{x}\right|^{2}\right|_{0} ^{l}\right\}+s \iiint \varphi_{4 x}|u|^{2}-\int\left[\varphi_{3 x}|u|^{2}\right]_{0}^{l}-2 \iint \varphi_{x x}\left|u_{x}\right|^{2}\right) \\
& +\left.2 s \operatorname{Re} \int \varphi_{x x} u \overline{u_{x}}\right|_{0} ^{l}-6 s^{3} \iint\left(\varphi_{x}\right)^{2} \varphi_{x x}|u|^{2}+\left.2 s^{3} \int\left(\varphi_{x}\right)^{3}|u|^{2}\right|_{0} ^{l}+2 s^{3} \iint \varphi_{x x}\left|\varphi_{x}\right|^{2}|u|^{2} \\
= & -4 s \iint \varphi_{x x}\left|u_{x}\right|^{2}+s \iint \varphi_{4 x}|u|^{2}-4 s^{3} \iint\left(\varphi_{x}\right)^{2} \varphi_{x x}|u|^{2} \\
& \quad+\left.\int\left[2 s \varphi_{x}\left|u_{x}\right|^{2}+\left(-s \varphi_{3 x}+2 s^{3}\left(\varphi_{x}\right)^{3}\right)|u|^{2}+2 s \varphi_{x x} \operatorname{Re}\left(u \overline{u_{x}}\right)\right]\right|_{0} ^{l} .
\end{aligned}
$$

On the other hand,

$$
\begin{aligned}
I_{1}^{2}= & i \iint\left(2 s \varphi_{x} u_{x}+s \varphi_{x x} u\right) \overline{u_{t}}-i \iint\left(2 s \varphi_{x} \overline{u_{x}}+s \varphi_{x x} \bar{u}\right) u_{t} \\
= & -i \iint\left(2 s \varphi_{x t} u_{x}+2 s \varphi_{x} u_{x t}+s \varphi_{x x t} u+s \varphi_{x x} u_{t}\right) \bar{u} \\
& \quad+i \iint 2 s\left(\varphi_{x x} u_{t}+\varphi_{x} u_{x t}\right) \bar{u}-\left.i \int 2 s \varphi_{x} \bar{u} u_{t}\right|_{0} ^{l}-i \iint s \varphi_{x x} \bar{u} u_{t} \\
= & -i \iint\left(s \varphi_{x x t}|u|^{2}+2 s \varphi_{x t} u_{x} \bar{u}\right)-\left.i \int 2 s \varphi_{x} \bar{u} u_{t}\right|_{0} ^{l} \\
= & i \iint s \varphi_{x t}\left(u \overline{u_{x}}+u_{x} \bar{u}\right)-\left.i \int s \varphi_{x t}|u|^{2}\right|_{0} ^{l}-2 i s \iint \varphi_{x t} u_{x} \bar{u}-\left.i \int 2 s \varphi_{x} \bar{u} u_{t}\right|_{0} ^{l} \\
= & i \iint s \varphi_{x t}\left(u \overline{u_{x}}-u_{x} \bar{u}\right)+\left.i \int\left[s \varphi_{x}\left(u \overline{u_{t}}-u_{t} \bar{u}\right)\right]\right|_{0} ^{l} .
\end{aligned}
$$

It remains to estimate $I_{2}$.

$$
I_{2}=\iint s \varphi_{t}\left(u \overline{u_{t}}+\bar{u} u_{t}\right)+\iint s \varphi_{t}\left(-i u \overline{u_{x x}}+i \bar{u} u_{x x}\right)=: I_{2}^{1}+I_{2}^{2}
$$


We find that

$$
\begin{aligned}
I_{2}^{1}= & -\iint s \varphi_{t t}|u|^{2}, \\
I_{2}^{2}= & i s \iint\left(\varphi_{x t} u+\varphi_{t} u_{x}\right) \overline{u_{x}}-\left.i s \int \varphi_{t} u \overline{u_{x}}\right|_{0} ^{l} \\
& \quad-i s \iint\left(\varphi_{t x} \bar{u}+\varphi_{t} \overline{u_{x}}\right) u_{x}+\left.i s \int \varphi_{t} \bar{u} u_{x}\right|_{0} ^{l} \\
= & 2 \operatorname{Re} \iint(i s) \varphi_{x t} u \overline{u_{x}}+\left.2 \operatorname{Re} \int(-i s) \varphi_{t} u \overline{u_{x}}\right|_{0} ^{l} .
\end{aligned}
$$

Thus

$$
\begin{aligned}
& I_{1}+I_{2}+I_{3} \\
& =-4 s \iint \varphi_{x x}\left|u_{x}\right|^{2}+s \iint \varphi_{4 x}|u|^{2}-4 s^{3} \iint\left|\varphi_{x}\right|^{2} \varphi_{x x}|u|^{2} \\
& \quad+i \iint s \varphi_{x t}\left(u \overline{u_{x}}-u_{x} \bar{u}\right)-s \iint \varphi_{t t}|u|^{2}+2 \operatorname{Re} \iint(i s) \varphi_{t x} u \overline{u_{x}} \\
& +\int\left[2 s \varphi_{x}\left|u_{x}\right|^{2}+\left(-s \varphi_{3 x}+2 s^{3}\left(\varphi_{x}\right)^{3}\right)|u|^{2}+2 s \varphi_{x x} \operatorname{Re}\left(u \overline{u_{x}}\right)\right. \\
& \left.\quad+i s \varphi_{x}\left(u \overline{u_{t}}-u_{t} \bar{u}\right)+2 \operatorname{Re}\left\{(-i s) \varphi_{t} u \overline{u_{x}}\right\}\right]\left.\right|_{0} ^{l} .
\end{aligned}
$$

We conclude that (with the indices written again)

$$
\begin{aligned}
\sum_{1 \leq k \leq N}\left\|\mathbf{w}^{k}\right\|^{2}=\sum_{1 \leq k \leq N} & {\left[\left\|M_{1}^{k} \mathbf{u}^{k}\right\|^{2}+\left\|M_{2}^{k} \mathbf{u}^{k}\right\|^{2}\right] } \\
& +\sum_{1 \leq j, k \leq N}\left\{-4 s \int_{0}^{T} \int_{0}^{l_{j}} \varphi_{j, x x}^{k}\left|u_{j, x}^{k}\right|^{2}-4 s \operatorname{Im} \int_{0}^{T} \int_{0}^{l_{j}} \varphi_{j, x t}^{k} u_{j}^{k} \overline{u_{j, x}^{k}}\right. \\
& +\int_{0}^{T} \int_{0}^{l_{j}}\left|u_{j}^{k}\right|^{2}\left[s\left(\varphi_{j, 4 x}^{k}-\varphi_{j, t t}^{k}\right)-4 s^{3}\left(\varphi_{j, x}^{k}\right)^{2} \varphi_{j, x x}^{k}\right] \\
& +\int_{0}^{T}\left[2 s \varphi_{j, x}^{k}\left|u_{j, x}^{k}\right|^{2}+\left(-s \varphi_{j, 3 x}^{k}+2 s^{3}\left(\varphi_{j, x}^{k}\right)^{3}\right)\left|u_{j}^{k}\right|^{2}+2 s \varphi_{j, x x}^{k} \operatorname{Re}\left(u_{j}^{k} \overline{u_{j, x}^{k}}\right)\right. \\
& \left.\left.+2 s \varphi_{j, t}^{k} \operatorname{Re}\left(-i u_{j}^{k} \overline{u_{j, x}^{k}}\right)+i s \varphi_{j, x}^{k}\left(u_{j}^{k} \overline{u_{j, t}^{k}}-u_{j, t}^{k} \overline{u_{j}^{k}}\right)\right]\left.\right|_{0} ^{l}\right\} .
\end{aligned}
$$


Step 2. Estimation of the boundary terms at the internal node $\mathcal{O}$.

We estimate each term in

$$
\begin{aligned}
& \sum_{j, k}(-2 s) \int_{0}^{T} \varphi_{j, x}^{k}(0)\left|u_{j, x}^{k}(0)\right|^{2}+\sum_{j, k} \int_{0}^{T}\left(s \varphi_{j, 3 x}^{k}(0)-2 s^{3}\left(\varphi_{j, x}^{k}(0)\right)^{3}\right)|\mathbf{u}(0)|^{2} \\
& +\sum_{j, k}(-2 s) \int_{0}^{T} \varphi_{j, x x}^{k}(0) \operatorname{Re}\left(\mathbf{u}(0) \overline{u_{j, x}^{k}(0)}\right)+\sum_{j, k}(-2 s) \int_{0}^{T} \varphi_{j, t}^{k}(0) \operatorname{Re}\left(-i \mathbf{u}(0) \overline{u_{j, x}^{k}(0)}\right) \\
& +\sum_{j, k} \int_{0}^{T}(-i s) \varphi_{j, x}^{k}(0)\left(\mathbf{u}(0) \overline{\mathbf{u}_{t}(0)}-\mathbf{u}_{t}(0) \overline{\mathbf{u}(0)}\right)=: J_{1}+J_{2}+J_{3}+J_{4}+J_{5} .
\end{aligned}
$$

In the above equation and in the following ones, we write merely

$$
\mathbf{u}(0):=u_{j}^{k}(0, t), \quad \varphi(0)=\varphi_{j}^{k}(0, t), \quad \text { etc. }
$$

Using (4.42), (4.43), and (4.33) (for the $q_{j}$ 's) we see that

$$
\begin{aligned}
J_{1}= & (-2 s) \sum_{j} \int_{0}^{T} \sum_{k} \varphi_{j, x}^{k}(0)\left|e^{-s \varphi(0)}\left(-s \varphi_{j, x}^{k} q_{j}+q_{j, x}\right)\right|^{2} \\
= & -2 s^{3} \int_{0}^{T} \sum_{j, k}\left(\varphi_{j, x}^{k}(0)\right)^{3}|\mathbf{u}(0)|^{2}-2 s \sum_{j} \int_{0}^{T}\left(\sum_{k} \varphi_{j, x}^{k}(0)\right) e^{-2 s \varphi(0)}\left|q_{j, x}(0)\right|^{2} \\
& \quad+4 s^{2} \operatorname{Re} \sum_{j} \int_{0}^{T}\left(\sum_{k}\left[\varphi_{j, x}^{k}(0)\right]^{2}\right) \mathbf{u}(0) \overline{q_{j, x}(0)} \\
= & -2 s^{3} \int_{0}^{T} \sum_{j, k}\left(\varphi_{j, x}^{k}(0)\right)^{3}|\mathbf{u}(0)|^{2} .
\end{aligned}
$$

Therefore

$$
J_{1}+J_{2}=\sum_{j, k} \int_{0}^{T}\left(s \varphi_{j, 3 x}^{k}(0)-4 s^{3}\left(\varphi_{j, x}^{k}(0)\right)^{3}\right)|\mathbf{u}(0)|^{2} .
$$

On the other hand, using (4.43), (4.44) and (4.33), we have that

$$
\begin{aligned}
J_{3}= & -2 s \sum_{j, k} \int_{0}^{T} \varphi_{j, x x}^{k}(0) \operatorname{Re}\left[\mathbf{u}(0) e^{-s \varphi(0)}\left(-s \varphi_{j, x}^{k}(0) \overline{q_{j}(0)}+\overline{q_{j, x}(0)}\right)\right] \\
= & 2 s^{2} \operatorname{Re} \int_{0}^{T}\left(\sum_{j, k} \varphi_{j, x x}^{k}(0) \varphi_{j, x}^{k}(0)\right)|\mathbf{u}(0)|^{2} \\
& +2 s \lambda \operatorname{Re} \int_{0}^{T} \frac{\mathbf{u}(0) e^{-s \varphi(0)} e^{\lambda \boldsymbol{\psi}(0)}}{t(T-t)} \sum_{j}\left(\sum_{k}\left(\psi_{j}^{k}\right)^{\prime \prime}(0)+\lambda \sum_{k}\left[\left(\psi_{j}^{k}\right)^{\prime}(0)\right]^{2}\right) \overline{q_{j, x}(0)} \\
= & 2 s^{2} \operatorname{Re} \int_{0}^{T}\left(\sum_{j, k} \varphi_{j, x x}^{k}(0) \varphi_{j, x}^{k}(0)\right)|\mathbf{u}(0)|^{2} .
\end{aligned}
$$


Combining (4.52), (4.53) and (4.45), we obtain that for $s \geq s_{1}$ and $\lambda \geq \lambda_{1}$,

$$
J_{1}+J_{2}+J_{3} \geq c s^{3} \lambda^{3} \sum_{j, k} \int_{0}^{T}\left(\frac{e^{\lambda \psi_{j}^{k}(0)}}{t(T-t)}\right)^{3}|\mathbf{u}(0)|^{2} .
$$

Finally, we claim that $J_{4}=J_{5}=0$. Indeed, using (4.33), we obtain that

$$
\begin{aligned}
J_{4} & =-2 s \operatorname{Im}\left(\int_{0}^{T} \varphi_{t}(0) u(0) \sum_{j, k} \overline{u_{j, x}^{k}(0)} d t\right) \\
& =-2 s \operatorname{Im} \int_{0}^{T} \varphi_{t}(0) u(0) \sum_{j, k}\left(-s \varphi_{j, x}^{k}(0) \overline{q(0)}+\overline{q_{j, x}(0)}\right) e^{-s \varphi(0)} d t \\
& =0,
\end{aligned}
$$

while $J_{5}=0$ by (4.41). Thus we conclude that

$$
J_{1}+J_{2}+J_{3}+J_{4}+J_{5} \geq c s^{3} \lambda^{3} \int_{0}^{T}\left(\frac{e^{\lambda \psi(0)}}{t(T-t)}\right)^{3}|\mathbf{u}(0)|^{2} .
$$

for $s \geq s_{1}, \lambda \geq \lambda_{1}$.

Step 3. Estimation of the integrals along the edges.

Direct estimations as in [30, Proposition 2.1] (without any integration by parts) yield that for some constant $A>0$

$$
\begin{aligned}
\sum_{j, k}\{( & -4 s) \int_{0}^{T} \int_{0}^{l_{j}} \varphi_{j, x x}^{k}\left|u_{j, x}^{k}\right|^{2}-4 s \operatorname{Im} \int_{0}^{T} \int_{0}^{l_{j}} \varphi_{j, x t}^{k} u_{j}^{k} \overline{u_{j, x}^{k}} \\
& \left.+\int_{0}^{T} \int_{0}^{l_{j}}\left|u_{j}^{k}\right|^{2}\left[s\left(\varphi_{j, 4 x}^{k}-\varphi_{j, t t}^{k}\right)-4 s^{3}\left(\varphi_{j, x}^{k}\right)^{2} \varphi_{j, x x}^{k}\right]\right\} \\
\geq & A \sum_{j, k}\left\{\lambda^{2} s \int_{0}^{T} \int_{0}^{l_{j}} \frac{e^{\lambda \psi_{j}^{k}}}{t(T-t)}\left|\left(\psi_{j}^{k}\right)^{\prime} u_{j, x}^{k}\right|^{2}+\lambda s^{3} \int_{0}^{T} \int_{0}^{l_{j}}\left|\varphi_{j, x}^{k}\right|^{3}\left|u_{j}^{k}\right|^{2}\right\}
\end{aligned}
$$

provided that $s \geq s_{2}, \lambda \geq \lambda_{2}$. Combining (4.51), (4.55) and (4.56), we infer that

$$
\begin{aligned}
& \sum_{j, k}\left\{\int_{0}^{T} \int_{0}^{l_{j}}\left[\left|\left(M_{1}^{k} \mathbf{u}^{k}\right)_{j}\right|^{2}+\left|\left(M_{2}^{k} \mathbf{u}^{k}\right)_{j}\right|^{2}\right]+\lambda^{2} s \int_{0}^{T} \int_{0}^{l_{j}} \frac{e^{\lambda \psi_{j}^{k}}}{t(T-t)}\left|\left(\psi_{j}^{k}\right)^{\prime} u_{j, x}^{k}\right|^{2}+\lambda s^{3} \int_{0}^{T} \int_{0}^{l_{j}}\left|\varphi_{j, x}^{k}\right|^{3}\left|u_{j}^{k}\right|^{2}\right. \\
& \left.+c s^{3} \lambda^{3} \int_{0}^{T}\left(\frac{e^{\lambda \psi(0)}}{t(T-t)}\right)^{3}|u(0)|^{2}\right\} \leq c \sum_{j, k}\left(\int_{0}^{T} \int_{0}^{l_{j}}\left|w_{j}^{k}\right|^{2}+s \int_{0}^{T}\left|\varphi_{j, x}^{k}\left(l_{j}\right)\right|\left|u_{j, x}^{k}\left(l_{j}\right)\right|^{2} d t\right) .
\end{aligned}
$$

Replacing $u_{j}^{k}$ by $e^{-s \varphi_{j}^{k}} q_{j}$ in (4.57) gives (4.46).

Remark 4.2. Note that (4.46) is still valid if, in the definition of $Z$, one replaces

$$
q_{j} \in C^{2,1}\left(\left[0, l_{j}\right] \times[0, T]\right) \quad \forall j \in[[1, N]]
$$


by

$$
\mathbf{q} \in H^{2,1}(\Gamma \times(0, T)) .
$$

4.2. The boundary problem. We consider the following boundary initial-value problem

$$
\begin{cases}i u_{j, t}+u_{j, x x}+p_{j}(x) u_{j}=0, & x \in\left(0, l_{j}\right), j \in[[1, N]], t \in(0, T), \\ u_{j}(0, t)=u_{l}(0, t), & j, k \in[[1, N]], t \in(0, T), \\ \sum_{1 \leq j \leq N} u_{j, x}(0, t)=0, & t \in(0, T), \\ u_{j}\left(l_{j}, t\right)=h_{j}(t), & j \in[[1, N]], t \in(0, T), \\ \mathbf{u}(x, 0)=\mathbf{u}_{0}(x), & x \in \Gamma\end{cases}
$$

In what follows we fix the initial data $\mathbf{u}_{0}$ and the boundary data $\mathbf{h}=\left\{h_{j}\right\}_{j=1, N}$, and we denote by $\mathbf{u}(\mathbf{p})$ the solution of the system (4.58) associated with the potential $\mathbf{p} \in L^{\infty}(\Gamma)$.

Theorem 4.3. Assume that $\mathbf{p} \in L^{\infty}(\Gamma ; \mathbb{R}), \mathbf{u}_{0} \in L^{\infty}(\Gamma)$ and $r>0$ are such that

- $\mathbf{u}_{0}(x) \in \mathbb{R}$ or $i \mathbf{u}_{0}(x) \in \mathbb{R}$ a.e. in $\Gamma$,

- $\left|\mathbf{u}_{0}(x)\right| \geq r>0$ a.e. in $\Gamma$, and

- $\partial_{t} \mathbf{u}(\mathbf{p}) \in H^{2,1}(\Gamma \times(0, T))$.

Then, for any $m \geq 0$, there exists a constant $C=C\left(m,\left\|\partial_{t} \mathbf{u}(\mathbf{p})\right\|_{H^{2,1}(\Gamma \times(0, T))}, r\right)>0$ such that for any $\mathbf{q} \in B_{m}(0) \subset L^{\infty}(\Gamma ; \mathbb{R})$ satisfying

$$
\partial_{t} \mathbf{u}(\mathbf{q}) \in H^{2,1}(\Gamma \times(0, T)),
$$

we have that

$$
\|\mathbf{p}-\mathbf{q}\|_{L^{2}(\Gamma)} \leq C \sum_{1 \leq j \leq N}\left\|\partial_{x}[\mathbf{u}(\mathbf{p})-\mathbf{u}(\mathbf{q})]_{j}\left(l_{j}, .\right)\right\|_{H^{1}(0, T)} .
$$

Proof. Pick any $\mathbf{p}, \mathbf{q}$ as in the statement of the theorem, and introduce the difference $\mathbf{y}:=$ $\mathbf{u}(\mathbf{p})-\mathbf{u}(\mathbf{q})$ of the corresponding solutions of (4.58). Then $y$ fulfills the system

$$
\begin{cases}i y_{j, t}+y_{j, x x}+q_{j}(x) y_{j}=f_{j}(x) R_{j}(x, t), & x \in\left(0, l_{j}\right), j \in[[1, N]], t \in(0, T), \\ y_{j}(0, t)=y_{l}(0, t), & j, k \in[[1, N]], t \in(0, T), \\ \sum_{1 \leq j \leq N} y_{j, x}(0, t)=0, & t \in(0, T), \\ y_{j}\left(l_{j}, t\right)=0, & j \in[[1, N]], t \in(0, T), \\ y(x, 0)=0, & x \in \Gamma,\end{cases}
$$

with $f_{j}=q_{j}-p_{j}$ (real valued) and $R_{j}:=(\mathbf{u}(\mathbf{p}))_{j}$. To complete the proof of Theorem 4.3, we need the following result.

Proposition 4.4. Suppose that $\mathbf{R}=\left\{R_{j}\right\}_{j=1, N}$ satisfies

- $\mathbf{R}(x, 0) \in \mathbb{R}$ or $i \mathbf{R}(x, 0) \in \mathbb{R}$ a.e. in $\Gamma$,

- $|\mathbf{R}(x, 0)| \geq r>0$ a.e. in $\Gamma$,

- $\mathbf{R} \in H^{1}\left(0, T ; L^{\infty}(\Gamma)\right)$, and

- $\partial_{t} \mathbf{y} \in H^{2,1}(\Gamma \times(0, T))$.

Then for any $m \geq 0$ there exists a constant $C=C\left(m,\left\|\mathbf{R}_{t}\right\|_{L^{2}\left(0, T ; L^{\infty}(\Gamma)\right)}, r\right)$ such that for any $\mathbf{q} \in L^{\infty}(\Gamma, \mathbb{R})$ with $\|\mathbf{q}\|_{L^{\infty}(\Gamma)} \leq m$ and for all $\mathbf{f} \in L^{2}(\Gamma, \mathbb{R})$, the solution $\mathbf{y}$ of (4.59) satisfies

$$
\|\mathbf{f}\|_{L^{2}(\Gamma)} \leq C \sum_{1 \leq j \leq N}\left\|y_{j, x}\left(l_{j}, .\right)\right\|_{H^{1}(0, T)}
$$


Proof of Proposition 4.4. Let $\mathbf{f} \in L^{2}(\Gamma ; \mathbb{R})$ and $\mathbf{R} \in H^{1}\left(0, T ; L^{\infty}(\Gamma)\right)$ be such that $\mathbf{R}(x, 0) \in \mathbb{R}$ a.e. in $\Gamma$, and let $\mathbf{y}$ be the solution of (4.59). We take the even-conjugate extensions of $\mathbf{y}$ and $\mathbf{R}$ to the interval $(-T, T)$; i.e., we set $\mathbf{y}(x, t)=\overline{\mathbf{y}(x,-t)}$ for $t \in(-T, 0)$ and similarly for $\mathbf{R}$. Since $\mathbf{R}(x, 0) \in \mathbb{R}$ a.e. in $\Gamma$, we have that $\mathbf{R} \in H^{1}\left(-T, T ; L^{\infty}(\Gamma)\right)$, and $\mathbf{y}$ satisfies the system (4.59) in $\Gamma \times(-T, T)$. In the case when $\mathbf{R}(x, 0) \in i \mathbb{R}$ a.e. in $\Gamma$, the proof is still valid by taking odd-conjugate extensions.

Let $\mathbf{z}(x, t)=\mathbf{y}_{t}(x,-t)$. Then $\mathbf{z}$ satisfies the following system:

$$
\begin{cases}z_{j, t}+i z_{j, x x}+i q_{j}(x) z_{j}=i f_{j}(x) R_{j, t}(x, t), & x \in\left(0, l_{j}\right), j \in[[1, N]], t \in(-T, T), \\ z_{j}(0, t)=z_{l}(0, t), & j, k \in[1, N]], t \in(-T, T), \\ \sum_{1 \leq j \leq N} z_{j, x}(0, t)=0, & t \in(-T, T), \\ z_{j}\left(l_{j}, t\right)=0, & j \in[1, N]], t \in(-T, T), \\ \mathbf{z}(x, 0)=-i \mathbf{f}(x) \mathbf{R}(x, 0), & x \in \Gamma .\end{cases}
$$

We apply Proposition 4.1 , but on the time interval $(-T, T)$ instead of $(0, T)$. Therefore, here we consider

$$
\theta_{j}^{k}(x, t)=\frac{e^{\lambda \psi_{j}^{k}(x)}}{(T+t)(T-t)}, \quad \varphi_{j}^{k}(x, t)=\frac{e^{\lambda C}-e^{\lambda \psi_{j}^{k}(x)}}{(T+t)(T-t)}, \quad \forall(x, t) \in \Gamma \times(-T, T) .
$$

As in the proof of Proposition 4.1, we introduce $\left.w_{j}^{k}=e^{-s \varphi_{j}^{k}} z_{j}, \widetilde{\left(M_{2}^{k}\right.} \mathbf{z}\right)_{j}=e^{s \varphi_{j}^{k}}\left(M_{2}^{k} \mathbf{w}^{k}\right)_{j}$ and $\left(M_{2}^{k} \mathbf{w}^{k}\right)_{j}=w_{j, t}^{k}+i\left(w_{j, x x}^{k}+s^{2}\left|\varphi_{j, x}^{k}\right|^{2} w_{j}^{k}\right)$. Next, we set

$$
\left.J=\sum_{1 \leq j, k \leq N} \int_{-T}^{0} \int_{0}^{l_{j}} e^{-2 s \varphi_{j}^{k}} \widetilde{\left(M_{2}^{k}\right.} \mathbf{z}\right)_{j} \overline{z_{j}} d x d t
$$

Then we have

$$
\begin{aligned}
J & =\sum_{j, k} \int_{-T}^{0} \int_{0}^{l_{j}}\left(M_{2}^{k} \mathbf{w}^{k}\right)_{j} \overline{w_{j}^{k}} d x d t \\
& =\sum_{j, k}\left\{\int_{-T}^{0} \int_{0}^{l_{j}} w_{j, t}^{k} \overline{w_{j}^{k}} d x d t+i \int_{-T}^{0} \int_{0}^{l_{j}}\left(-\left|w_{j, x}^{k}\right|^{2}+s^{2}\left|\varphi_{j, x}^{k}\right|^{2}\left|w_{j}^{k}\right|^{2}\right) d x d t+\left.i \int_{-T}^{0} w_{j, x}^{k} \overline{w_{j}^{k}}\right|_{0} ^{l_{j}} d t\right\} .
\end{aligned}
$$

Note that, by (4.61) and (4.41),

$$
\sum_{j} w_{j, x}^{k}(0) \overline{w_{j}^{k}(0)}=\sum_{j}\left(z_{j, x}(0)-s \varphi_{j, x}^{k}(0) z(0)\right) e^{-2 s \varphi(0)} \overline{\mathbf{z}(0)}=0 .
$$

Therefore

$$
\operatorname{Re}(J)=\frac{1}{2} \sum_{j, k} \int_{0}^{l_{j}}\left|w_{j}^{k}(x, 0)\right|^{2} d x d t=\frac{1}{2} \sum_{j, k} \int_{0}^{l_{j}} e^{-2 s \varphi_{j}^{k}(x, 0)}|\mathbf{f}(x)|^{2}|\mathbf{R}(x, 0)|^{2} d x .
$$

Using the hypothesis on $\mathbf{R}(x, 0)$, we infer that

$$
\operatorname{Re}(J) \geq \frac{r^{2}}{2} \sum_{j, k} \int_{0}^{l_{j}} e^{-2 s \varphi_{j}^{k}(x, 0)}|\mathbf{f}(x)|^{2} d x .
$$


On the other hand, we have that

$$
\begin{aligned}
|J| & \left.\left.\leq\left.\sum_{j, k}\left\{\left(\int_{-T}^{0} \int_{0}^{l_{j}} e^{-2 s \varphi_{j}^{k}} \mid \widetilde{M_{2}^{k}} \mathbf{z}\right)_{j}\right)\right|^{2} d x d t\right)^{\frac{1}{2}}\left(\int_{-T}^{0} \int_{0}^{l_{j}} e^{-2 s \varphi_{j}^{k}}\left|z_{j}\right|^{2} d x d t\right)^{\frac{1}{2}}\right\} \\
& \left.\leq\left.\frac{1}{2} \sum_{j, k}\left\{\lambda^{-2} s^{-\frac{3}{2}} \int_{-T}^{0} \int_{0}^{l_{j}} e^{-2 s \varphi_{j}^{k}} \mid \widetilde{\left(M_{2}^{k}\right.} \mathbf{z}\right)_{j}\right|^{2} d x d t+\lambda^{2} s^{\frac{3}{2}} \int_{-T}^{0} \int_{0}^{l_{j}} e^{-2 s \varphi_{j}^{k}}\left|z_{j}\right|^{2} d x d t\right\} \\
& \left.\leq\left. c \lambda^{-2} s^{-\frac{3}{2}} \sum_{j, k}\left\{\int_{-T}^{0} \int_{0}^{l_{j}} e^{-2 s \varphi_{j}^{k}} \mid \widetilde{\left(M_{2}^{k}\right.} \mathbf{z}\right)_{j}\right|^{2} d x d t+\lambda^{4} s^{3} \int_{-T}^{0} \int_{0}^{l_{j}}\left(\theta_{j}^{k}\right)^{3} e^{-2 s \varphi_{j}^{k}}\left|z_{j}\right|^{2} d x d t\right\},
\end{aligned}
$$

where we used the fact that

$$
\theta_{j}^{k} \geq T^{-2}
$$

From (4.63), the Carleman estimate (4.46) (applied on the interval $(-T, T)$ instead of $(0, T)$ ), and the fact that $\varphi_{j}^{k}(x, 0) \leq \varphi_{j}^{k}(x, t)$ for all $(x, t) \in\left(0, l_{j}\right) \times(-T, T)$, that $\theta_{j}^{k} e^{-2 s \varphi_{j}^{k}}$ is bounded from above in $\left(0, l_{j}\right) \times(-T, T)$, that $\mathbf{q} \in L^{\infty}(\Gamma)$, and that $\mathbf{R}_{t} \in L^{2}\left(-T, T ; L^{\infty}(\Gamma)\right)$, we infer that for $s$ and $\lambda$ large enough

$$
\begin{aligned}
|J| & \leq c \lambda^{-2} s^{-\frac{3}{2}} \sum_{j, k}\left\{\int_{-T}^{T} \int_{0}^{l_{j}} e^{-2 s \varphi_{j}^{k}}\left|\mathbf{f} \mathbf{R}_{t}\right|^{2} d x d t+\lambda s \int_{-T}^{T} \theta_{j}^{k} e^{-2 s \varphi_{j}^{k}}\left|z_{j, x}\left(l_{j}, t\right)\right|^{2} d t\right\} \\
& \leq c \lambda^{-2} s^{-\frac{3}{2}} \sum_{j, k} \int_{0}^{l_{j}} e^{-2 s \varphi_{j}^{k}(x, 0)}|\mathbf{f}|^{2} d x+c \lambda^{-1} s^{-\frac{1}{2}} \sum_{j} \int_{-T}^{T}\left|z_{j, x}\left(l_{j}, t\right)\right|^{2} d t .
\end{aligned}
$$

It follows from (4.62), (4.64), and the fact that $\mathbf{z}(x, t)=-\overline{\mathbf{z}(x,-t)}$ for $(x, t) \in \Gamma \times(-T, 0)$, that for $s$ and $\lambda$ large enough

$$
\sum_{j, k} \int_{0}^{l_{j}} e^{-2 s \varphi_{j}^{k}(x, 0)}|\mathbf{f}(x)|^{2} d x \leq c \sum_{j} \int_{-T}^{0}\left|z_{j, x}\left(l_{j}, t\right)\right|^{2} d t .
$$

Then (4.60) follows from (4.65) since

$$
e^{-2 s \varphi_{j}^{k}(x, 0)} \geq e^{-2 s T^{-2}\left(e^{\lambda C}-1\right)} .
$$

This completes the proof of Proposition 4.4 and of Theorem 4.3.

\section{Open PROBLEMS}

We now mention a few open problems related to our work. One of them is whether it is possible to reduce the number of measurements at the boundaries. It could be interesting to combine the ideas of the paper with those appearing in [13], [14] where less measurements on the boundary are needed but some rationality assumptions on the lengths of the edges have to be made. For the Schrödinger equation, the question whether a Carleman estimate on a tree with $N$ exterior vertices can be written with only one weight function and $N-1$ boundary observations seems to be challenging. 
The extension of the present work to more general graphs with other kind of coupling is also an open problem. We recall here the works of Kostrykin and Schrader [23, 24] where self-adjoint Laplace operators with general coupling conditions are introduced.

\section{ACKNOWLEDGEMENTS}

The authors wish to thank Institut Henri Poincaré (Paris, France) for providing a very stimulating environment during the "Control of Partial Differential Equations and Applications" program in the Fall 2010. LI is supported by the grants PCE-2011-3-0075, "Analysis, control and numerical Approximations of PDE" and TE-4/2010 of CNCSIS Romania. LR is partially supported by the Agence Nationale de la Recherche, Project CISIFS, grant ANR-09-BLAN0213-02. AP is partially supported by CNPq, Math-Amsud project CIP-PDE, and French-Brasil agreement.

\section{REFERENCES}

[1] S. Alexander. Superconductivity of networks. A percolation approach to the effects of disorder. Phys. Rev. $B$ (3), 27(3):1541-1557, 1983.

[2] S. Avdonin, G. Leugering, and V. Mikhaylov. On an inverse problem for tree-like networks of elastic strings. ZAMM Z. Angew. Math. Mech., 90(2):136-150, 2010.

[3] L. Baudouin, E. Crépeau and J. Valein. Global Carleman estimate on a network for the wave equation and application to an inverse problem, preprint (hal-00576296), 2011.

[4] L. Baudouin, A. Mercado. An inverse problem for Schrödinger equations with discontinuous main coefficient. Appl. Anal. Vol. 87, no. 10-11, 11451165, 2008.

[5] L. Baudouin, A. Mercado, A. Osses. A global Carleman estimate in a transmission wave equation and application to a one-measurement inverse problem. Inverse Problems Vol. 23, no. 1, 257278, 2007.

[6] L. Baudouin and J.-P. Puel. Uniqueness and stability in an inverse problem for the Schrödinger equation. Inverse Problems, 18(6):1537-1554, 2002.

[7] A. Benabdallah, Y. Dermenjian and J. Le Rousseau. Carleman estimates for the one-dimensional heat equation with a discontinuous coefficient and applications to controllability and an inverse problem. J. Math. Anal. Appl. Vol. 336, no. 2, 865887, 2007.

[8] A. L. Bukhgeĭm and M. V. Klibanov. Uniqueness in the large of a class of multidimensional inverse problems. Dokl. Akad. Nauk SSSR, 260(2):269-272, 1981.

[9] L. Cardoulis, M. Cristofol, P. Gaitan. Inverse problem for the Schrödinger operator in an unbounded strip. J. Inverse Ill-Posed Probl. vol. 16, no. 2, 127146, 2008.

[10] L. Cardoulis, P. Gaitan. Simultaneous identification of the diffusion coefficient and the potential for the Schrödinger operator with only one observation. Inverse Problems Vol. 26, no. 3, 035012, 10 pp., 2010.

[11] C. Cattaneo. The spectrum of the continuous Laplacian on a graph. Monatsh. Math., 124(3):215-235, 1997.

[12] J. P. Carini, J. T. Londergan, D. P. Murdock, D. Trinkle and C. S. Young. Bound states in waveguides and bent quantum wires. I. Applications to waveguide systems, Phys. Rev. B, 55:9842-9851, 1997.

[13] R. Dáger. Observation and control of vibrations in tree-shaped networks of strings. SIAM J. Control Optim., 43(2):590-623 (electronic), 2004.

[14] R. Dáger and E. Zuazua. Wave propagation, observation and control in 1-d flexible multi-structures, volume 50 of Mathématiques \& Applications (Berlin) [Mathematics \& Applications]. Springer-Verlag, Berlin, 2006.

[15] P. Exner. Vertex coupling in quantum graphs: approximations by scaled Schrödinger operators, to appear in Proceedings of the ICM satellite conference "Mathematics in Science and Technology" New Delhi, August 15-17, 2010.

[16] A. Figotin and Y. A. Godin. Spectral properties of thin-film photonic crystals. SIAM J. Appl. Math., 61(6):1959-1979 (electronic), 2001. 
[17] A. V. Fursikov and O. Yu. Imanuvilov. Controllability of evolution equations, volume 34 of Lecture Notes Series. Seoul National University Research Institute of Mathematics Global Analysis Research Center, Seoul, 1996.

[18] O. Yu. Imanuvilov and Masahiro Yamamoto. Global uniqueness and stability in determining coefficients of wave equations. Comm. Partial Differential Equations, 26(7-8):1409-1425, 2001.

[19] V. Isakov. Carleman type estimates in an anisotropic case and applications J. Diff. Eqns $10521738,1993$.

[20] V. Isakov. Inverse Problems for Partial Differential Equations (Berlin: Springer), 1998.

[21] M. V. Klibanov. Inverse problems in the "large" and Carleman estimates. Differential Equations, 20(6):755760, 1984.

[22] M. 1. V. Klibanov. Inverse problems and Carleman estimates. Inverse Problems, 8(4):575-596, 1992.

[23] V. Kostrykin and R. Schrader. Kirchhoff's rule for quantum wires. J. Phys. A, 32(4):595-630, 1999.

[24] V. Kostrykin and R. Schrader. Laplacians on metric graphs: eigenvalues, resolvents and semigroups. In Quantum graphs and their applications, volume 415 of Contemp. Math., pages 201-225. Amer. Math. Soc., Providence, RI, 2006.

[25] P. Kuchment. Graph models for waves in thin structures. Waves Random Media, 12(4):R1-R24, 2002.

[26] P. Kuchment. Quantum graphs: an introduction and a brief survey. In Analysis on graphs and its applications, volume 77 of Proc. Sympos. Pure Math., pages 291-312. Amer. Math. Soc., Providence, RI, 2008.

[27] P. Kuchment. Quantum graphs. I. Some basic structures. Waves Random Media, 14(1):S107-S128, 2004. Special section on quantum graphs.

[28] P. Kuchment and O. Post. On the spectra of carbon nano-structures. Comm. Math. Phys., 275(3):805-826, 2007.

[29] I. Lasiecka, R. Triggiani and X. Zhang. Global uniqueness, observability and stabilization of nonconservative Schrödinger equations via pointwise Carleman estimates: Part I. H1-estimates J. Inv. Ill-posed Problems 11 43123, 2004.

[30] A. Mercado, A. Osses, and L. Rosier. Inverse problems for the Schrödinger equation via Carleman inequalities with degenerate weights. Inverse Problems, 24(1):015017, 18, 2008.

[31] J.-P. Puel and M. Yamamoto. Generic well-posedness in a multidimensional hyperbolic inverse problem. J. Inverse Ill-Posed Probl., 5(1):55-83, 1997.

[32] L. Rosier and B.-Y. Zhang. Null controllability of the complex Ginzburg-Landau equation. Ann. Inst. H. Poincaré Anal. Non Linéaire, 26(2):649-673, 2009.

[33] M. Yamamoto. Uniqueness and stability in multidimensional hyperbolic inverse problems. J. Math. Pures Appl. (9), 78(1):65-98, 1999.

[34] M. Yamamoto, Carleman estimates for parabolic equations and applications, Inverse problems 25 (2009) $123013(75 \mathrm{pp})$

(L. I. Ignat) Institute of Mathematics "Simion Stoilow" of the Romanian Academy, 21 Calea Grivitei Street, 010702 Bucharest, Romania

AND

BCAM - Basque Center for Applied Mathematics, Bizkaia Technology Park, Building 500 Derio, Basque Country, Spain.

E-mail address: liviu.ignat@gmail.com

(A. F. Pazoto) Instituto de Matemática, Universidade Federal do Rio de Janeiro, P.O. Box 68530, CEP 21945-970, Rio DE JANEIRO, RJ, BRASIL

E-mail address: ademir@im.ufrj.br

(L. Rosier) Institut Elie CARTAN, UMR 7502 UHP/CNRS/INRIA, B.P. 70239, 54506 VANDCEUVRE-LÈSNancy Cedex, France

E-mail address: rosier@iecn.u-nancy.fr 